\title{
Une bibliographie sur la translocation 1/29 de bovins dans le monde (1964-1990)
}

\author{
CP Popescu 1, A Pech 2 \\ ${ }^{1}$ INRA - CRJ, laboratoire de cytogénétique; \\ 2 INRA - CRJ, département de génétique animale, 78352 Jouy-en-Josas Cedex, France
}

(Reçu le 26 août 1991; accepté le 21 octobre 1991)

\begin{abstract}
Résumé - Cette synthèse présente les références des 231 articles publiés de 1964 à 1990 sur là translocation $1 / 29$ chez les bovins. Plusieurs tableaux et figures indiquent ensuite le nombre des publications par année et pays, la distribution géographique de l'anomalie sur la carte du globe et sa fréquence par race. Dans les 2 dernières parties, on trouve l'index race, pays et l'index auteurs.
\end{abstract}

bovin / translocation $1 / 29$

Summary - Cattle $1 / 29$ translocation in the world (1964-1990): a review. This paper presents a reference list of 231 articles published between 1964 and 1990 on the 1/29 translocation in cattle. Several tables and figures show the number of papers per year and country and the geographic distribution of this abnormality around the world. Another table presents the frequency of the heterozygous carriers for each breed. The last two chapters consist of a breed/country index and an author index.

cattle / 1/29 translocation

La translocation Robertsonienne $1 / 29$ a été signalée pour la première fois en 1964, en Suède par I Gustavsson. Depuis, elle a été retrouvée dans de nombreux pays sur les 5 continents.

En raison de ses effets négatifs sur la fertilité, par augmentation de la mortalité embryonnaire chez les porteurs hétérozygotes, beaucoup de pays ont mis en place des laboratoires d'analyse cytogénétique de routine et ont adopté une politique d'éradication. La recherche systématique de cette anomalie, en particulier chez les taureaux d'insémination artificielle, a fait du bœuf domestique l'espèce de mammi- fères la plus étudiée du point de vue cytogénétique, après l'homme et la souris.

Nous avons pensé qu'une bibliographie qui se veut exhaustive, pour la période 1964-1990, sur cette anomalie, serait utile pour tous ceux qui travaillent sur la cytogénétique de cette espèce.

Dans une première partie, sont présentées les références des 231 articles par ordre alphabétique des auteurs. Ensuite, 2 figures indiquent le nombre de publications par année (1) et par pays (2). La figure 3 représente la distribution de l'anomalie sur la mappemonde. Le tableau I montre les fréquences des porteurs hété- 
rozygotes par race et le tableau $\|$, par pays.

Les fréquences par race signalées dans le tableau I ont été calculées dans certains cas sur les résultats d'une étude rigoureusement menée. Dans d'autres cas, les résultats ont été obtenus sur un échantillonnage qui n'est pas forcément aléatoire et de ce fait, les fréquences calculées ne peuvent être considérées comme représentatives pour une race donnée.

Pour la même raison, nous n'avons pas calculé une fréquence moyenne par race. Les cas signalés représentent des situations où on ne connaît pas le nombre d'animaux examinés. On doit enfin remarquer que dans certains cas, bien que la fréquence calculée représente les animaux porteurs hétérozygotes, elle peut inclure aussi des porteurs homozygotes.

Les 2 dernières parties contiennent un index race/pays et un index auteurs.

Si l'on estime à environ un millier, les publications existantes à ce jour sur la cytogénétique des grands mammifères d'élevage, 231 , soit près du quart sont consacrées à cette seule anomalie.

\section{RÉFÉRENCES}

001 Amrud J (1969) Centric fusion of chromosomes in Norwegian red cattle. Hereditas 62 , 293-302. MC * : Norvège / bovin / translocation 1/29 / Pie-Rouge (Simmental) / fertilité femelle

002 Arruga MV, Monteagudo LV, Lopez N, Catalan J (1990) A 60, XY/59, XX t 1/29 mosaic presumptively chimeric in a case of questionable pseudohermaphrodite bull. In: IXth Eur Coll Cytogenet Domest Anim.ToulouseAuzeville, 7-13 Juillet, 21, poster. MC : Espagne / bovin / translocation 1/29

003 Arruga MV, Zarazaga I (1984) Frequency distribution of the Robertsonian translocation $(1 / 29)$ in cattle populations bred in Spain. In: Vith Eur Coll Cytogenet Domest Anim. Zu- rich, 16-20 Juillet 1984, 43-51. MC : Espagne / bovin / Pie Noire (Holstein Frisonne) / Pirenaica / Lidia / Normande / Maine Anjou / Pie Rouge (Simmental), Charolais / Vianesa, Caldelatan / Limiana / Alistana / Sayaguesa / Mirandesa / Cachena / Morucha / Retinta / translocation 1/29

004 Arruga MV, Zarazaga I (1985) Chromosome studies in Spanish fighting bulls. Arch Zootec 34 (128), 49-65. MC : Espagne / bovin / taureau de combat / translocation 1/29

005 Arruga MV, Zarazaga I (1987) La translocation Robertsoniana 1/29 en el ganado vacuno. Su incidencia en las razas vacunas espanolas. Genet lber 39, 61-75. MC : Espagne / bovin / translocation 1/29

006 Arruga MV, Zarazaga I, Vallejo M (1984) (Structural chromosome anomalies in cattle: centric fusion (1/29) and inversion (25)) (espagnol) An Fac Vet Leon (18-19), 163-164. MC : Espagne / bovin / Pie Noire (Holstein Frisonne) / Translocation 1/29

007 Arruga MV, Zarazaga I, Vallejo M, Burguette I (1984) The 1/29 Robertsonian translocation in autochthonous Spanish cattle breed: hereditary studies in families. In: VIth Eur Coll Cytogenet Domest Anim, Zurich, 16-20 Juillet 1984, 129-136. MC : Espagne / bovin / translocation 1/29 / races espagnole

008 Begimkulov BK, Bakai AV, Krasota VF (1980) A Robertsonian type translocation in a Zebu bull and two of his sons. DokJ Vses Akad S-Kh Nauk, (11), 31-33. MC : URSS / bovin / zébu / translocation 1/29

009 Berardino D (di), lannuzzi L, Ferrara L, Matassino D (1980) Cytogenetic survey on Podolian cattle reared in Southern Italy. I. Inventory of chromosome aberrations. In: IVth Eur Coll Cytogenet Domest Anim, Uppsala, 1013 juin 1980, 142-151. MC : Italie / bovin / Podolian / translocation $1 / 29$

010 Bergant J, Locniskar F (1979) (Karyotypes of bulls at the Al centre at Preska) Slovaque rés angl). $Z b$ Bioteh Fak Univ Ljublj, Kmetijstvo, 34, 71-109. MC : Tchécoslovaquie / bovin / Brune des Alpes (Brown Swiss) / croisement / translocation 1/29

011 Betancourt A (1982) (C-bands in Santa Gertrudis cows with the $1 / 29$ translocation) (esp) Rev Salud Anim 4, 129-136. MC : Cuba / bovin / Santa Gertrudis / translocation 1/29 
012 Betancourt A (1983) Serum characters and fertility of Santa Gertrudis bulls with the 1/29 translocation. Rev Salud Anim 5, 149-158. MC : Cuba / bovin / Santa Gertrudis / translocation $1 / 29$

013 Betancourt A (1985) Fertility data from daughters of a Santa Gertrudis bull with chromosomal translocation 1/29. Beitr Trop Landwirstsch Veterinarmed 23 (1), 77-80. MC : Cuba / Santa Gertrudis / translocation 1/29 / fertilité femelle

014 Betancourt A, Gutierrez C, Sanchez A (1982) Efecto de la fusion centrica 1/29 sobre la fertilidad de la raza Santa Gertrudis (Effect of the 1/29 centric fusion on fertility in the Santa Gertrudis breed). Rev Salud Anim 4, 127-132. MC : Cuba / croisement / Santa Gertrudis / translocation 1/29 / fertilité mâle / mortalité embryonnaire

015 Betancourt A, Gutierrez C, Sanchez A (1985) Chromosomal translocation $1 / 29$ in Santa Gertrudis sires. Beitr Trop Landwintsch Veterinartmed, 23 (1), 73-75. MC : Cuba / bovin / translocation 1/29 / Pie Noire (Holstein Frisonne) / Santa Gertrudis / zébu

016 Betancourt A, Sanchez A, Gutierrez C (1978) Centric fusion in Santa Gertrudis breed. In: XIV Int Congr Genet Moscow, 2130 August 1978, Part 1, 500. MC : Cuba/ bovin / Santa Gertrudis / translocation 1/29

017 Blazak WF, Eldridge FE (1977) A Robertsonian translocation and its effect upon fertility in Brown Swiss cattle. J Dairy Sci 60, 11331142. MC : Etats-Unis / bovin / Brune des Alpes (Brown Swiss) / translocation 1/29 / fertilité femelle

018 Bongso A, Basrur PK (1976) Chromosome anomalies in Canadian Guernsey bulls. Cornell Vet 66, 476-489. MC : Canada / bovin / Guernsey / Translocation 1/29 / fertilité mâle

019 Bouvet A, Cribiu EP (1990) Synaptonemal complex behaviour in a bull homozygous for the 1/29 Robertsonian translocation. Genet Sel Evol 22, 361-366. MC : France / bovin / Blonde d'Aquitaine / translocation 1/29

020 Bruere AN, Chapman HM (1973) Autosomal translocations in two exotic breeds of cattle in New Zealand. Vet Rec 92, 615-620. MC : Nouvelle Zélande / bovin / translocation / Pie Rouge (Simmental) / Blonde d'Aquitaine / Limousin / Charolais / Maine-Anjou
021 Buoen LC, Weber AF, Meiske JC, Hooker EC (1988) Cases of 1/29 Robertosonian translocation (Centric fusion) in Charolais cattle. Can Vet J 29, 455-457. MC : ÉtatsUnis / bovin / Charolais / translocation 1/29

022 Ciupercescu DD (1980) (Studies on frequency and effects of the $1 / 29$ and 14/20 robertsonian translocations in Simmental cattle from Transylvania) (roum rés angl). Bul Inst Agron Cluj-napoca 34, 99-103. MC : Roumanie / bovin / Pie Rouge (Simmental) / translocation 1/29 / translocation 14/20 / fertilité femelle

023 Ciupercescu DD (1980) Cytogenetic studies on Simmental bulls used for artificial insemination in Romania (roum rés angl) in: Simp "Probl Amelior Teh Crestere Patol Taurine Ovine", 16-17 mai 1980, Cluj-Napoca, 5761. MC : Roumanie / bovin / Pie Rouge (Simmental) / translocation 1/29

024 Ciupercescu DD (1982) Distribution of the $1 / 29$ and 14/20 Robertsonian translocation in Romanian Simmental (Romanian Spotted) sires (rés esp). In: 2nd World Congr Genet Appl Livest Prod 4-8 octobre 1982, vol 8, 135-141, Editorial Garsi, Madrid. MC : Roumanie / bovin / Pie Rouge (Simmental) / translocation $1 / 29$

025 Ciupercescu DD, Sabau T (1983) Observatii asupra fecunditatii taurilor cu translocatii Robertsoniene $1 / 29$ si $14 / 20$ (Fertility of bulls with the $1 / 29$ and 14/20 Robertsonian translocations). Lucrarile celui de al 8 lea Semin Amelior Technol Patol Rumegatoarelor, Cluj-Napoca 11-12 noiembrie 1983, 71-74. MC : Roumanie / bovin / Pie Rouge (Simmental) Translocation 1/29/fertilité

026 Cribiu EP, Popescu CP (1977) L'aneuploïdie et la polyploïdie chez les bovins dans les cultures de cellules bovines. Ann Génét Sél Anim 9, 275-282. MC : France / bovin / translocation 1/29 / chromosome / Charolais / Limousin / Maine-Anjou / Normande / Pie Noire (Holstein Frisonne)

027 Cribiu EP, Popescu CP (1978) Étude des anomalies caryotypiques. Elev Insémination (165), 21-23. MC : France / bovin / translocation $1 / 29$

028 Cribiu EP, Popescu CP (1979) Étude des anomalies caryotypiques. Elev Insémination (170), 5-6. MC : France / Côte d'lvoire / bovin / translocation 1/29 / N'Dama / Baoule / zébu soudanais 
029 Cribiu EP, Popescu CP (1980) La cytogénétique au service de l'élevage. Effet de la fusion centrique. Rec Méd Vét 156, 319-322. MC : France / bovin / translocation 1/29 / fertilité femelle / bibliographie

030 Dain AR, Dott HM (1985) Cytogenetic studies of 1-29 translocation carrying cows and their embryos. Theriogenology 23 (4), 641 653. MC : Grande Bretagne / bovin / translocation $1 / 29$ / Pie Rouge (Simmental) / Charolais / embryon

031 Dain AR, Dott HM, Newcomb R, Schwabe AE (1985) Cytogenetic studies of 1-29 translocation-carrying cows and their embryos. Theriogenology 23, 641-653. MC : Grande Bretagne / bovin / translocation 1/29 / embryon

032 Darre R, Gary F, Berland HM, Concordet D (1990) Frequency and effects of the 1/29 Robertsonian translocation in Blonde d'Aquitaine breed. In: IXh Eur. Coll Cytogenet Domest Anim Toulouse-Auzeville, 7-13 July 1990, 24 abstr. MC : France / bovin / Blonde d'Aquitaine / translocation 1/29

033 Darre R, Queinnec G, Berland HM (1972) La translocation $1 / 29$ des bovins. Étude générale et importance du phénomène dans le Sud-Ouest. Rev Méd Vét 123, 477-494. MC : France / Limousin / Charolais / Blonde d'Aquitaine / translocation 1/29

034 Dyrendahl I (1971) Fertiliteten hos tjurar med kromosomenbbining (Fertility in bulls with a chromosome deficiency). Husdjur 5 , 20. MC : Suède / bovin / Pie Rouge (Simmental) / fertilité mâle / fusion chromosomique

035 Dyrendahl I, Gustavsson I (1979) Sexual functions, semen characteristics and fertility of bulls carrying the $1 / 29$ chromosome translocation. Hereditas 90, 281-289. MC : Suède / Pie Rouge (Simmental) / translocation 1/29 / fertilité mâle / fertilité femelle

036 Eldridge FE (1975) High frequency of a Robertsonian translocation in a herd of Britisch White cattle. Vet Rec 96, 71-73. MC : Grande Bretagne / Blanche Britannique (British White) / translocation 1/29

037 Eldridge FE, Balakrishnan CR (1977) Cband variations in Robertsonian transloca- tions in cattle. Nucleus 20, 28-30. MC : ÉtatsUnis / bovin / translocation 1/29

038 Eldridge FE, Blazak WF (1975) A chromosomal aberration in Brown Swiss cattle. In: XVIlth Annu Meet Am Dairy Sci Assoc, Kansas State University, Manhattan, 22-25 juin 1975. J Dairy Sci 58, 756 (abstr). MC : ÉtatsUnis / Brune des Alpes (Brown Swiss) / translocation 1/29 / anomalie chromosomique / bibliographie

039 Eldridge FE, Harris NB, Koenig JLF (1984) Chromosomes of young Al bulls. Vith Eur Coll Cytogenet Domest Anim, Zurich, Suisse, July 16-20, 1984, 59-67. MC : États-Unis / bovin /Brune des Alpes (Brown Swiss) I translocation 1/29 / anomalie chromosomique / bibliographie

040 Falaschini AF, Codeluppi S, Ferriani AM, Morea R (1984) Indagine sulla traslocazione 1/29 nella razza bovina Romagnola (The 1/29 translocation in Romagna cattle). Atti Soc Ital Sci Vet 38, 407-410. MC : Italie / bovin / Romagnola / transiocation 1/29

041 Fechheimer NS (1972) Chromosome abnormalities and embryo death in farm animals. Vet Rec (26), 241-243. MC : États-Unis / animal domestique / anomalie chromosomique / fertilité / transiocation

042 Fechheimer NS (1973) A cytogenetic survey of young bulls in the USA. Vet Rec 93, 535536. MC : États-Unis / bovin / translocation 1/ 29 / bovin laitier / Ayrshire / Guernesey / Pie Noire (Holstein Frisonne) / Jersey / Brune des Alpes (Brown Swiss) / bovin à viande / Aberdeen Angus / Charolais / Hereford / Rouge sans cornes (Red Poll)

043 Ferrungem Moraes JC, Pitta Pinheiro JE, Poli JLEH, Vaz AK, Risch ALC (1985) Constituiçao cromossomica em bovinos com fenotipo normal e portadores de malformaçoes diversas (Chromosomal status in normal cattle and animals carrying different malformations). Rev Bras Reprod Anim 9, 75-96. MC : Brésil / bovin / translocation 1/29 / anomalie chromosomique

044 Fischer H (1971) Beitrag zurkenustruis des einheimischen thailandischen Rindes mit Hilfe der Chromosomenanalyse. Z Tierz Zuechtungsbiol 88, 215-221. MC : Thaïlande / bovin / Thaï / fusion centrique

* MC : Mots clés 
045 Fischer H, Hohn H, Schevrmann E (1975) Untersuchungen uber die karyotypen des Braunen Atlas Rindes. Glessener Beitr Erbpath Zuchthyg 6, 70-79. MC : Maroc / Brune de l'Atlas / translocation 1/29

046 Forster M (1980) Chromosome constitution as a factor in fertility, 31st Annual Meeting Eur Assoc Anim Prod, 7 p. MC : Allemagne / bovin / translocation 1/29 / fertilité

047 Forster M, Helm W, Stranzinger G (1980) Analyse cytogénétique de populations sur des bovins bavarois. Zuchtungskunde 52, 417-420. MC : Allemagne / bovin / Bavarois / translocation 1/29 / chimérisme / fertilité femelle

048 Forster M, Stranzinger G (1975) Boviden als zusamdengehorige Translocationsstramme (Bovids as related translocationstrains). Z Tierzucht ZuchtBiol 92, 267-271. MC : Allemagne / bovin / translocation

049 Foulley JL, Frebling J (1983) Note sur la translocation 1/29 en races bovines à viande françaises. INRA, Station de Génétique quantitative et appliquée, Jouy-en-Josas, 20 p. MC : France / bovin / translocation 1/29 / bibliographie / fertilité mâle / fertilité femelle

050 Foulley $\mathrm{JL}$, Frebling $\mathrm{J}$ (1985) Critères de détection indirecte des taureaux porteurs de la translocation 1/29 à partir des caryotypes de leurs descendants. Génét Sél Evol 17 (3), 341-350. MC : France / translocation 1/29/ bovin / critères de détection / théorème de Bayes

051 Foulley JL, Frebling J (1985) La translocation 1/29 chez les bovins: distribution, effets, procédure d'éradication. Bull Tech CRZV Theix, INRA (62), 93-102. MC : France / bovin / translocation 1/29 / éradication / reproduction femelle / reproduction mâle

052 Franck M, Laurent C, Froget J, Surcin JM, Coursat F (1980) Chromosomal abnormalities and fertility of carriers. The 1/29 Robertsonian translocation. IVth Eur Coll Cytogenet Domest Anim Uppsala, 10-13 juin 1980 , 55-62. MC : France / bovin / translocation 1/ 29 / fertilité mâle / fertilité femelle

053 Frebling J, Foulley JL, Berland HM, Popescu CP, Cribiu EP, Darre R (1987) Résultats de l'enquête sur la fréquence de la translocation $1 / 29$ en race bovine Blonde d'Aquitaine. Bull Tech CRZV, Theix INAA (67), 49-58. MC :
France / bovin / Blonde d'Aquitaine / translocation $1 / 29$

054 Froget J, Coulon J, Nain MC, Dalbiel JM (1972) Anomalie chromosomique de type fusion centrique chez un veau Charolais. Bull Soc Sci Vét Méd Comp Lyon, 74, 131-135. MC : France / Charolais / bovin / fusion centrique

055 Giovanni AM (de), Molteni L (1976) Ultérieure étude cytogénétique chez la race bovine Romagnola. Note 1, Nouveaux cas de fusion centrique. Atti Soc Ital Sci Vet 30, 479 481. MC : Italie / translocation 1/29/Romagnola

056 Giovanni AM (de), Succi G, Molteni L (1977) Cytogenetic study on some Italian cattle populations underexploited or almost extinguished. Atti Soc Ital Sci Vet 30, 581-582. MC : Italie / bovin / Rendena / Modicana / Reggiana / translocation 1/29

057 Goldman IL, Bakai AV, Zhivalev IK (1974) Translocation (Centrical Confluence) of Robertson's type autosomes in cattle (Bos taurus L). Tsitol Genet 8, 548-557. MC : URSS / bovin / translocation

058 Goldman IL, Dun EA, Bakai AV (1979) (A 1/ 29 chromosome translocation in a calf of the black Friesian breed) (rus rés angl). Tsitol Genet 13, 28-30. MC : URSS / bovin / Pie Noire (Holstein Frisonne) / translocation 1/29

059 Graphodatsky AS, Sharshov AA, Kulikova SG (1990) Novel chromosome abberations in cattle. IXth Eur Coll Cytogenet Domest Anim, Toulouse-Auzeville, 7-13 July 1990, 25 poster. MC : URSS / bovin / translocation 1/29

060 Guanti G, Minoia RG (1978) A Robertsonian translocation of the female cells of a bull, cotwin to a free-martin. Cornell Vet 68 (1), 94 97. MC : Italie / bovin / Brune des Alpes (Brown Swiss) / translocation 1/29 / fertilité

061 Gustafsson H, Larsson K, Gustavsson I (1985) Karyotypes and morphological and histological alterations of the genetic tracts of repeat breeder heifers with known breeding history. Acta Vet Scand 26, 1-12. MC : Suède / bovin / translocation 1/29 / Pie Rouge (Simmental) / fertilité femelle / mortalité embryonnaire

062 Gustavsson I (1966) Chromosome abnormality in cattle. Nature 211, 865-866. MC : 
Suède / bovin / translocation / Pie Rouge (Simmental)

063 Gustavsson I (1969) Cytogenetic distribution and phenotypic effects of a translocation in Swedish cattle. Hereditas 63, 68-169. MC : Suède / bovin / Pie Rouge (Simmental) / translocation $1 / 29$ / fertilité femelle

064 Gustavsson I (1970) Economic importance of a translocation in Swedish cattle. In: Eur Koll Zytogenet (Chromosomenpathologie) Veterinarmed Tierz Saugetierkde, Giessen, 12-13 oct 1970, 34-42. MC : Suède / bovin / Pie Rouge (Simmental) / translocation 1/29/ fertilité femelle / conséquence économique

065 Gustavsson I (1971) Culling rates in daughters of sires with a translocation of centric fusion type. Hereditas 67, 65-74. MC : Suède / bovin / Pie Rouge (Simmental) / translocation $1 / 29$ / fertilité femelle

066 Gustavsson I (1971) Chromosomes of repeat-breeder heifers. Hereditas 68, 331-332. MC : Suède / bovin / translocation 1/29 / fertilité femelle

067 Gustavsson I (1971) Distribution of the $1 / 29$ translocation in the Al bull population of Swedish Red and White cattle. Hereditas 69, 101-106. MC : Suède / bovin / Pie Rouge (Simmental) / translocation 1/29

068 Gustavsson I (1974) Appearance and persistence of the $1 / 29$ translocation in cattle. In: Coll "Les accidents chromosomiques de la reproduction 1973" INSERM, Paris, France, 147-153. MC : Suède / bovin / translocation 1/29 / bibliographie

069 Gustavsson I (1975) New information on the reduced fertility of cattle with the 1/29 translocation. In: 2 Eur Koll Zytogenet (Chromosomenpathologie) Veterinarmed Tierz Saugetierkde, Giessen, 184-188, Dtsch Veterinarmed Ges. MC : Suède / bovin / Pie Rouge (Simmental) / translocation $1 / 29 /$ fertilité mâle

070 Gustavsson I (1975) Some comments on eradication of the 1/29 translocation in Sweden (cattle). 2. Eur Koll Zytogenet (Chromosomenpathologie) Veterinarmed Tierz Saugetierkde, Giessen, 263-268, Dtsch Veteri, larmed Ges. MC : Suède / bovin / translocation 1/29 / fertilité mâle

071 Gustavsson I (1979) Distribution and effects of the 1/29 Robertsonian translocation in cattle. J Dairy Sci 62, 825-835. MC : Suède / bovin / fertilité mâle / bibliographie / translocation 1/29, Pie Rouge (Simmental)

072 Gustavsson I (1980) (Chromosome aberrations and their influence on the reproductive performance of domestic animals - a review). (all rés angl fr esp). $Z$ Tierz Zuchtungsbiol 97, 176-195). MC : Suède / anomalie chromosomique / animal domestique / bibliographie / porcin / bovin / cheval / ovin / translocation / mortalité embryonnaire / fertilité

073 Gustavsson I (1984) Chromosome evaluation and fertility. Xth Int Congr Anim/ Reprod Artif Insemination, Univ Illinois, UrbanaChampaign, USA, June 10-14, 1984, 6, 1-7. MC : Suède / bibliographie / bovin / porcin / fusion centrique / translocation

074 Gustavsson I (1985) Experience of the eradication work for eliminating the 1/29 translocation from the Swedish Red and White cattle breed. Am Dairy Sci Assoc VIIIth Annu Meet Supplement June 9-12, 1985, University of Illinois, Urbana Champaign, Supplement 1, 1985, J Dairy Sci, 68, 248 (abstr). MC : Suède / bovin / Pie Rouge (Simmental) / translocation 1/29 / eradication / fertilité

075 Gustavsson I, Hageltorn M (1976) Staining technique for definite identification of individual cattle chromosomes in routine analysis. $J$ Hered 67, 175-178. MC : Suède / bovin / translocation 1/29 / technique de bandes

076 Gustavsson I, Hageltorn M, Zech L (1976) Identification of the $1 / 29$ translocation in the Swedish Red and White (SRB) cattle breed by utilization of new staining techniques. Hereditas 82, 260-262. MC : Suède / bovin / Pie Rouge (Simmental) / translocation 1/29

or7 Gustavsson I, Rendel J (1971) A translocation in cattle and its association to polymorphisms in red cell antigens, transferrins and carbonic anhydrases. Hereditas 67, 35-38. MC : Suède / bovin / Pie Rouge (Simmen(al) / translocation

078 Gustavsson I, Rockborn G (1964) Chromosome abnormality in three cases of lymphatic leukaemia in cattle. Nature 203, 990. MC : Suède / bovin / translocation / Pie Rouge (Simmental)

079 Halnan CRE (1976) A cytogenetic survey of 1101 Australian cattle of 25 differents breeds. Ann Génét Sél Anim 8, 131-139. MC : Aus- 
tralie / bovin / Hereford / Pie Noire (Holstein Frisonne) / Aberdeen Angus / caryotype / culard / translocation robertsonienne

080 Halnan CRE (1982) The paradox of the $Y$ chromosome and fertility in cattle. Vth Eur Coll Cytogenet Domest Anim Proc Milano, June 7-11 1982, 71-83. MC : Australie / bovin / fertilité / Hereford / Banteng

081 Halnan CRE, Galloway DB, Watson Jl, Mckee JJ, Norman J (1980) An autosomal centric fusion translocation in the Red Poll Breed in Australia. Vet Rec 107, 177-178. MC : Australie / bovin / Rouge sans cornes (Red Poll) / translocation 1/29 / fertilité mâle

082 Hanada H, Muramatsu S, Abe T, Fukushima $T$ (1981) Robertsonian chromosome polymorphism found in a local herd of Japanese Black cattle. Ann Génét Sél Anim 13, 205211. MC : Japon / bovin / Noire Japonaise (Japanese Black) / translocation

083 Hansen KM, Elleby F (1975) Chromosome investigation of Danish Al beef bulls. Nord Vet Med 27, 102-106. MC : Danemark / bovin / Charolais / Pie Rouge (Simmental), Hereford / Limousin / translocation

O84 Hansen KM, Hansen EM (1990) The 1/29 chromosome translocation in Danish Blonde d'Aquitaine cattle. IXth Eur Coll Cytogenet Domest Anim Toulouse-Auzeville, 7-13 Juillet 1990, 9, abstr. MC : Danemark / bovin / Blonde d'Aquitaine / translocation 1/29

085 Hari JJ, Franceschi P, Casabianca F, Boscher J, Popescu CP (1984) Étude cytogénétique d'une population de bovins corses. $C A$ Séances Acad Agric Fr 70, 191-199. MC : France / Corse / bovin / translocation 1/29

086 Harris EJ, Weiermans JE, Nel ND, Meyer EHH (1984) An investigation of chromosomal abnormalities in certain Southern African cattle breeds. In: Proc 2nd World Congr sheep beef cattle breeding, CSIR Conf Cent Pretoria 16-19 april 1984, 2, 1-5. MC : Afrique du Sud / bovin / Brahman / Bonsmara / Nguni / Charolais $x$ Jersey / translocation / caryotype

087 Harvey MJA (1971) An autosomal translocation in the Charolais breed of cattle. Vet Rec 89, 110-111. MC : Grande Bretagne / bovin / Charolais / translocation 1/29

088 Harvey MJA (1972) Chromosome abnormalities of cattle in Britain. Vet Rec 91, 630. MC : Grande Bretagne / bovin / translocation 1/29
089 Harvey MJA (1972) Chromosome analysis of cattle in Great Britain. Proc VII Int Congr Anim Reprod Artif Insemination, Munich 1101-1103. MC : Grande-Bretagne / bovin / Limousin / Pie Rouge (Simmental) / translocation $1 / 29 /$ fertilité femelle

090 Harvey MJA (1974) Chromosome analysis of cattle populations. Vet Rec 94, 227. MC : Grande Bretagne / bovin / translocation 1/29 / bibliographie

091 Harvey MJA (1976) Veterinary cytogenetics. Vet Rec 98, 479-481. MC : GrandeBretagne / bovin / Pie Noire (Holstein Frisonne) / Charolais / Pie Rouge (Simmental)

092 Herschler MS, Fechheimer NS (1966) Centric fusion of chromosomes in a set of bovine triplets. Cytogenetics 5, 307-312. MC : ÉtatsUnis / bovin / fusion centrique

093 Herzog A, Hohn H (1971) (Cytogenetic analysis of calves with congenital abnormalities of the central nervous system) (all rés angl). Ann Génét Sél Anim 3, 225-234. MC : Allemagne / bovin / translocation / Pie Rouge (Simmental)

094 Herzog A, Hohn H, Rieck GW (1977) Survey of recent situation of chromosome pathology in different breeds of German cattle. Ann Génét Sél Anim 9, 471-491. MC : Allemagne / bovin / translocation 1/29 / bibliographie

095 Hohn H (1971) (Autosomal chromosome translocation (centromere fusion) in heterosexual bovine twins) (all) Giessener Beitr Erbpathol Zuchthyg 3, 7-17. MC : Allemagne / bovin / Pie Rouge (Simmental) / translocation $1 / 29$ / fertilité

096 Hohn H, Herzog A (1975) Inheritance of Robertsonian translocation in two cow families with special reference to fertility conditions. 2 . Eur Koll Zytogenet (Chromosomenpathologie) Veterinarmed, Tierz Saugetierkde, Giessen, 189-192. MC : Allemagne / bovin / Pie Rouge (Simmental) / translocation 1/29

097 Igartua DV, Roldan ERS, Vitullo AD (1985) (Cytogenetic studies in beef cattle females with reproductive problems) (esp). Rev Argent Prod Anim 5. MC : Argentine / bovin / Aberdeen Angus / Hereford / créole / croisement

098 Institute of Animal Physiology, UK (1986) 1/ 29 translocation. Report for 1984 \& 1985, 95- 
96. MC : Grande-Bretagne / bovin / translocation 1/29 / Pie Rouge (Simmental)

099 Jaszczak K (1980) (Cytogenetic examinations of bulls from several chosen rearing centres) (pol rés angl rus) Pr Mater Zootech 22, 27-32. MC : Pologne / bovin / Pie Noire (Holstein Frisonne) / Pie Rouge (Simmental) / Charolais / caryotype / fusion centrique

100 Jaszczak K, Wijas B, Parada R (1990) (Cytogenetic examinations of bulls used in the animal breeding and insemination stations in Poland) (pol rés angl rus) Pr Mater Zootech (40) 67-74. MC : Pologne / bovin / Charolais / translocation $1 / 29$

101 Kachura VS (1987) (Chromosome aberrations in cattle - economic and evolutionary aspects). Vi S'ezd vses genetikov $i$ seleksionerov imienni NI, Vavilova, Moscou, 24-26 Tech Dokl T 3. Moscou, URSS, 95-96. MC : URSS / bovin / Pie Rouge (Simmental), Montbéliard / Lebedin / Pie Noire (Holstein Frisonne) / translocation 1/29

102 Kachura VS, Meleshko AA (1985) (La fréquence des translocations robertsoniennes chez les bovins d'Ukraine) (rus rés angl). Cyto/ Genet 19, 43-48. MC : URSS / bovin / Pie Rouge (Simmental) / Montbéliard / Lebedin / translocation 1/29 / chimérisme

103 King WA, Guay P (1982) La mortalité embryonnaire chez les animaux domestiques: aspects chromosomiques. Med Vet Quebec 12, 51-55. MC : Canata / bovin / porcin / ovin / cheval / mortalité embryonnaire / fertilité femelle / anomalie chromosomique / translocation / bibliographie

104 King WA, Linares $T$ (1983) A cytogenetic study of repeat-breeder heifers and their embryos. Can Vet J 24, 112-115. MC : Suède / bovin / Pie Rouge (Simmental) / Pie Noire (Holstein Frisonne) / croisement

105 King WA, Linares T, Gustavsson I (1981) Cytogenetics of preimplantation embryos sired by bulls heterozygous for the $1 / 29$ translocation. Hereditas 94, 219-224. MC : Suède / bovin / Pie Rouge (Simmental) / translocation $1 / 29$ / embryon

106 King WA, Linares T, Gustavsson I, Bane A (1980) Presumptive translocation type trisomy in embryos sired by bulls heterozygous for the 1/29 translocation. Hereditas 92, 167169. MC : Suède / bovin / translocation 1/ 29 / embryon
107 Kovacs A (1976) (A new autosomal translocation in Hungarian Simmentals) (all). 2. Eur Koll Zytogenet (Chromosomenpathologie) Veterinarmed Tierz Saugetierkde, Giessen, 29-30 september 1975, 162-167. MC : Hongrie / bovin / Pie Rouge (Simmental) / translocation $1 / 29$

108 Kovacs $A$ (1978) Occurrence of $1 / 29$ translocation in Hungarian Grey cattle. Eur Assoc Anim Prod XXIXth Annu Meet. Ann Genet Sel Anim 10, 594 (res). MC : Hongrie / bovin / Grise des steppes / translocation 1/ 29 / chimérisme

109 Kovacs A (1984) Progress in eradication of the 1/29 translocation of cattle in Hungary. VIth Eur Colloq Cytogenet Domest Anim Zurich, Suisse, July 16-20, 52-58. MC : Hongrie / bovin / Grise des steppes / Pie Rouge (Simmental) / Pie Noire (Holstein Frisonne) / translocation 1/29

110 Kovacs A, Meszaros I (1976) Centric fusion of the chromosome 1 and 29 in a Hungarian spotted bull. Anatomia Histologia Embryologia 5, 100-101. MC : Hongrie / bovin / Grise des steppes / translocation $1 / 29$

111 Kurchanov NA, Zhigachev Al (1989) (On the elimination of centromeric heterochromatin block on the arisal of Robertsonian translocation $1 / 29$ in cattle) (en russe). Sel' skokhoz Biol (2), 131-133. MC : URSS / bovin / translocation $1 / 29$ / hétérochromatine

112 Langhammer H (1986) Auswirkungen der 1/ 29 Translokation beim Fleischring (Effects of the 1/29 translocation in beef cattle). Tierzucht 40, 181-182. MC : Allemagne / bovin à viande / translocation 1/29 / insémination artificielle

113 Livescu BE, Samarineanu M, Granciu I, Patrascu M (1979) (The results of the first cytogenetic examination of the Al bulls in use in Romania) (roum, rés angl, fr all, rus). Lucr Stiint Inst Cercet Pentru Cresterea Taurinelor-Corbeanca 5, 19-30. MC : Roumanie / bovin / Pie Noire (Holstein Frisonne) / Brune des Alpes (Brown Swiss) / Charolais / chimérisme / translocation

114 Logue DN (1977) Meiosis in the domestic ruminants with particular reference to Robertsonian translocations. Ann Génét Sél Anim 9, 493-507. MC : Grande-Bretagne / ovin / bovin / caprin / translocation robertsonienne / méiose / cytogénétique 
115 Logue DN (1978) Chromosome banding studies in cattle. Res Vet Sci 25, 1-6. MC : Grande-Bretagne / bovin / Charolais / Rouge sans cornes (Red Poll) / Pie Rouge (Simmental) / translocation 1/29

116 Logue DN, Breeze RG (1977) Arthrogryposis-palatoschisis and a 1/29 translocation in a Charolais herd. Vet Rec 100, 509-510. MC : Grande-Bretagne / Charolais / translocation 1/29

117 Logue DN, Harvey MJA (1978) Meiosis and spermatogenesis in bulls heterozygous for a presumptive 1/29 Robertsonian translocation. $J$ Reprod Fert 54, 159-165. MC : GrandeBretagne / bovin / translocation 1/29 / méiose / fertilité mâle / Charolais

118 Lojda $L$ (1974) Centric chromosome fusion in Czech red spotted cattle. Veterinarstvi 24 (8), 342. Congr Cytogenet Giessen (1975) MC : Tchécoslovaquie / bovin / Pie Rouge (Simmental) / translocation 1/29

119 Lojda L (1976) Chromosome translocation 1/ 29 in Czechoslavakian Red spotted cattle. II. Europaisches Kolloquium uber Zytogenetik (Chromosomenpathologie) in Vetrinarmedizin, Tierzucht und Saugetierkunde, Giessen, 168-171. MC : Tchécoslovaquie / bovin / Pie Rouge (Simmental) / translocation 1/29

120 Lojda L, Rubes J, Stavikova M, Havrankova $J$ (1976) Chromosomal findings in some reproductive disorders in bulls. VIIIth Int Congr Anim Reprod Artif Insem; Krakow, July 1216. Proc Vol 1, 158 (abstr). MC : Tchécoslovaquie / bovin / Pie Rouge (Simmental) / translocation 1-29

121 Long SE (1985) Centric fusion translocations in cattle: a review. Vet Rec (116), 516-518. MC : Grande-Bretagne / bovin / translocation / bibliographie / races britanniques

122 Long SE (1989) The 1/29 centric fusion translocation in British White cattle in the UK. VIth $N$ Am Collog Cytogenet Domest Anim Purdue Univ, July 1989, P6. MC : GrandeBretagne / bovin / Blanche Britannique (British White) / translocation 1/29

123 Marx W (1979) (Des recherches cytogénétiques de la race Brune de l'Atlas au Maroc en considération spéciale de preuve des translocations 1 et 29) (all rés angl). Inaugural Dissertation zur Erlangung des Doktorgrades, Leibzig, Universitat zu Giessen, 58 p. MC :
Maroc / bovin / Brune de l'Atlas / translocation $1 / 29$

124 Marx W (1980) 1/29 Chromosomentranslokation beim Braunen Atlas Rind. Bend Munch Tierzucht Wschr 93, 264-266. MC : Maroc / bovin / Brune de l'Atlas / translocation $1 / 29$

125 Masuda H, Shioya Y, Fukuhara R (1980) Robertsonian translocation in Japanese Black cattle. Jap J Zootech Sci 51, 26-32. MC : Japon / bovin / Noire Japonaise (Japanese Black) / translocation 1/29 / translocation 5/ 21

126 Masuda H, Yasushi W (1980) Chromosome observations in a low fertility bull. Jap J Anim Res 2, 1-4. MC : Japon / bovin / fertilité mâle / anomalie chromosomique

127 Maurer RR, Vogt DW (1985) Decreased calving efficiency in females carrying the 1/29 chromosome translocation. Am Dairy Sci Assoc, VIIIth Annu Meet, June 9-12, Univ IIIinois, Urbana Champaign, (suppl 1). J Dairy Sci 68, 247 (abstr). MC : Etats-Unis / bovin / Pie Rouge (Simmental) / translocation 1/29 / reproduction femelle

128 Maurer RR, Vogt DW (1988) Decreased fertility in related females heterozygous for the $1 /$ 29 chromosome translocation. Theriogenology 30, 1149-1157. MC : États-Unis / bovin / Pie Rouge (Simmental) / translocation 1/29

129 Mayr B (1977) Demonstration of a Robertsonian translocation in the Austrian Simmental. Wiener Tierarztliche Monatsschrift 64, 186187. MC : Autriche / bovin / Brune des Alpes (Brown Swiss), Pie Rouge (Simmental)

130 Mayr B, Kopp E, Schleger W (1980) Evidence of Robertsonian 1/29 translocation in Oberintal Grey cattle (all rés angl). Wien tierarzt/ Mschr 67, 292-294. MC : Autriche / Grise Autrichienne (Tyrol Grey) / translocation 1/29

131 Mayr B, Schleger W (1977) Cytogenetic investigations incorporated in an Austrian programme for genetic prophylaxis in cattle (all rés angl ir rus). Zuchtungskde 49, 343-346. MC : Autriche / bovin / Pie Rouge (Simmental) / Brune des Alpes (Brown Swiss) / translocation 1/29

132 Mayr B, Schleger W (1978) The role of chromosome defects and variants in cattle breeding. XXe Reun annu FEZ, 5-7 juin 1978, 
Stockholm. Ann Génét Sél Anim 10, 593 (abstr). MC : Autriche / bovin / transiocation $1 / 29$

133 Meyer EHH, Harris EJ (1980) A Jersey $x$ Charolais bull with chromosome 1/29 translocation and testicular hypoplasia. Proc VIIth $S$ Afr Genetic Congr 2-8. MC : Afrique du Sud / bovin / translocation 1/29 / Jersey $\times$ Charolais / fertilité mâle

${ }^{134}$ Molteni L, Succi G (1976) (Étude cytogénétique ultérieure chez la race bovine Romagnola. Note 2. L'idiogramme de la race) (ital, rés angl, fr). Atti Soc It Sci Vet 30, 482-483. MC : Italie / bovin / Romagnola / cytogénétique

135 Moraes JCF, Mattevi MS, Salzano FM, Poli JLEH, Erdtmann B (1980) A cytogenetic survey of five breeds of cattle from Brazil. J Hered 71, 146-148. MC : Brésil / bovin / Charolais / Normandie / Pie Rouge (Simmental) / Santa Gertrudis / translocation / anomalie chromosomique / mosaïque

136 Morelli RH, Malheiros EB, Pinheiro LEL (1985) Avaliaçao fisico-morfologica dos espermatozoides de bovinos normais e de portadores da translocaçao Robertsoniana 1/29. (Physical and morphological evaluation of semen from normal bulls and carriers of the 1/29 Robertsonian translocation). Ars Vet 1 , 103-110. MC : Brésil / bovin / pitengueiras / translocation $1 / 29$, reproduction mâle

137 Moreno M, Delgado JV, Bonilla R (1985) Incidencia de la translocacion 1/29 en vacas de la raza Retinta con trastornos de la reproduccion. (Incidence of the 1/29 translocation in cows of Retinta breed with reproductive disturbs). Arch Zootec 34 (130), 211-217. MC : Espagne / bovin / Retinta / translocation $1 / 29$ / reproduction femelle

138 Moustafa AR, Ibrahim MAR, Rahman HA, Kovacs A (1983) Quality, freezability and fertility of the semen of pre-selected Al bulls carrying various chromosome aberrations. Anim Reprod Sci 6, 167-175. MC : Hongrie / bovin / Pie Noire (Holstein Frisonne) / Pie Rouge (Simmental) / translocation / chimerisme / fertilité mâle

139 McWhir J, Church RB, Coulter GH, Lin CC (1987) Incidence and inheritance of the $1 / 29$ and 14/20 Robertsonian translocations in $\mathrm{Ca}$ nadian beef cattle. Genome 29, 504-509. MC : Canada / bovin / Pie Rouge (Simmental) / translocation 1/29 / fertilité
140 Nevada VE, Burkat JT, Kachura VS / Meleshko AA, Amitrash NA, Leontieva IA (1983) (Result of cytogenetic control of breeding bulls at artificial insemination stations) (rus rés angl). Tsitol Genet 17, 34-38. MC : URSS / bovin / cytogénétique / Montbeliard / anomalie chromosomique / Chianina $\times$ Charolais $x$ Pie Rouge (Simmental)

141 Nel ND, Harris EJ, Scholtz MM, Lombard P (1984) An evaluation of the $1 / 29$ translocation heterozygote. IInd World Congr on sheep and beef cattle breed, Genetic research and indigeneous breeds exhibition 18 April, 9. MC : Afrique du Sud / bovin / Nguni / translocation $1 / 29$

$142 \mathrm{Nel} N D$, Harris EJ, Weiermans JE, Meyer EHH (1985) The 1/29 translocation in Southern African cattle. Am Dairy Sci Assoc VIIIth Annu Meet, June 9-12, Univ Illinois, Urbana Champaign, (suppl 1). J Dairy Sci68, 247 (abstr). MC : Afrique du Sud / bovin / races sud africaines / Nguni / Zébu Malawi / Pedi / Brahman / Charolais $x$ Jersey / translocation 1-29

$143 \mathrm{Nel}$ ND, Harris EJ, Weiermans JE, Meyer EHH (1988) The recent introduction of a $1 / 29$ chromosome translocation in South African Brahman cattle. Génét Sél Evol 20, 239-246. MC : Afrique du Sud / bovin / Brahman / translocation $1 / 29$

144 Nel ND, Harris EJ, Weiermans JE, Meyer $E H H$, Brix K (1985) A 1/29 chromosome translocation in Southern African Nguni cattle. The identification, occurrence and origin of the translocation. Génét Sél Evol 17 (3), 293-302. MC : Afrique du Sud / bovin / Nguni / translocation 1/29

145 Nosach VK, Avakova AG (1985) (Karyotyping of bulls) (en russe). Zhivotnovodstvo 11, 44-46. MC : URSS / bovin / caryotype

146 Papp M, Kovacs A (1978) Routine chromosomal examination of Al bulls in Hungary. $X X^{\theta}$ Réun annu FEZ, 5-7 juin, Stockholm. Ann Génét Sél Anim 10, 593 (abstr). MC : Hongrie / bovin / translocation 1/29 / Pie Rouge (Simmental) / Pie Noire (Holstein Frisonne) / Limousin / Hereford / Kostroma / croisés

147 Pathiraja N, Oyedipe EO, Buvanendran V (1985) Robertsonian translocation in a Zebu bull. Theriogenology 24 (4), 419-423. MC : Nigeria / Red Bororo / translocation 1/29 / fertilité mâle 
148 Pinheiro LEL (1979) (Cytogenetic studies in cattle: evolution and uses). A review (portug). Rev Bras Reprod Anim 1, 33-36. MC : Brésil / bovin / cytogénétique / bibliographie / translocation

149 Pinheiro LEL, Almeida IL, Garcia JM, Basrur PK (1987) Trisomy $X$ and $1 / 29$ translocation in infertile heifers. Theriogenology 28, 891898. MC : Brésil / bovin / Pitengueiras / translocation $1 / 29$

150 Pinheiro LEL, Ferrari I (1980) (A new type of Robertsonian translocation in cattle) (portug rés angl). Vie Encontró de Pesquisas Veterinarias, 6 e 7 de novembro de 1980, jaboticabal, Brazil, 93 (resumo). MC : Brésil / bovin / Pitengueiras / translocation 1/29

151 Pinheiro LEL, Ferrari I, Ferraz JBS, Lobo RB (1981) High frequency of Robertsonian translocation in a Brazilian cattle breed. Rev Bras Genet 4, 657-665. MC : Brésil / bovin / Rouge sans cornes (Red Poll) $\times$ Zebu (Pitangueiras) / translocation 1/29

152 Pinheiro LEL, Ferrari I, Lobo RB (1979) Robertsonian translocation in imported bulls utilized at artificial insemination centers in Brazil. Rev Brasil Genet 2, 135-143. MC : Brésil / bovin / Marchigiana / Pie Rouge (Simmental) / translocation 1/29

153 Pinheiro LEL, Waldrigues A, Ferrari I, Nogueira CP (1982) Characterization of the nucleolus organizing region in Zebu (Bos taurus indicus) and in animals with 1/29 centric fusion. Rev Brasil Genet 5, 551-560. MC : Brésil / bovin / zébu / translocation 1/29

154 Pollock DL (1974) Chromosome studies in artificial insemination sires in Great Britain. Vet Rec 95 (12), 266-267. MC : GrandeBretagne / bovin / translocation / fusion centrique / chimérisme

155 Popescu CP (1971) Deux cas nouveaux de fusion centrique chez les bovins (Note). Ann Génét Sél Anim 3, 521-526. MC : France / bovin / Montbéliard / translocation 1/29

156 Popescu CP (1973) L'hétérochromatine constitutive dans le caryotype bovin normal et anormal. Ann Génét 16, 183-188. MC : France / bovin / caryotype / fusion centrique

157 Popescu CP (1973) Nouvelles observations sur une fusion centrique chez Bos taurus $\mathrm{L}$. Ann Génét Sél Anim 5, 435-440. MC : France / bovin / Montbéliard / Charolais /
Blonde d'Aquitaine / Blonde d'Aquitaine $x$ Charolais / translocation 1/29

158 Popescu CP (1975) Sur la fréquence de la translocation $1 / 29$ dans certaines races bovines. II. Europaisches Kolloquium uber Zytogenetik (Chromosomenpathologie) in Veterinarmedizin, Tierzucht und Saugetierkunde, Giessen, 277-282, Dtsche veterinarmedi Ges. MC : France / bovin / translocation 1/29

159 Popescu CP (1975) L'étude du caryotype bovin (Bos taurus $L$ ) par les méthodes des bandes. Ann Biol Anim Biochim Biophys 15, 751-756. MC : France / bovin / caryotype / translocation $1 / 29$

160 Popescu CP (1976) Sur la fréquence de la translocation 1/29 dans certaines races bovines. II. Europaisches Kolloquium uber Zytogenetik (Chromosomenpathologie) in Veterinarmedizin, Tierzucht und Saugetierkunde, Giessen, 29-30 September, 277-282. MC : France / bovin / Charolais / Montbeliard / Limousin / Blonde d'Aquitaine

161 Popescu CP (1976) Observations sur le caryotype normal et anormal des bovins. Inf Vet 18, 2-4. MC : France / bovin / translocation $1 / 29$

162 Popescu CP (1976) Etudes cytogénétiques (anomalies caryotypiques). Elev Insém 153, 41-47. MC : France / bovin / translocation 1/ 29

163 Popescu CP (1977) Les anomalies chromosomiques des bovins (Bos taurus $\mathrm{L}$ ) : état actuel des connaissances. Ann Génét Sél Anim 9, 463-470. MC : France / bovin / anomalie chromosomique / translocation 1/29 / bibliographie

164 Popescu CP (1977) Anomalies chromosomiques et troubles de la fertilité. Elev Insem 159, 11-15. MC : France / Scandinavie / bovin / translocation 1/29 / bibliographie

165 Popescu CP (1978) Chromosome abnormalities and their consequences in domestic animals. $X X^{\theta}$ Réun annu FEZ, 5-7 juin, Stockholm. Ann Génét Sél Anim 10, 592-593 (abstr). MC : France / bovin / translocation 1/ $29 /$ méiose

166 Popescu CP (1978) A study of meiotic chromosomes in bulls carrying the $1 / 29$ translocation. Ann Biol Anim Biochim Biophys 18, 383-389. MC : France / bovin / translocation $1 / 29 /$ méiose 
167 Popescu CP (1980) Cytogenetic study on embryos sired by a bull carrier of 1/29 translocation. IVth Eur Collog Cytogenet Domest Anim, Uppsala, 182-186. MC : France / bovin / embryon / translocation 1/29

168 Popescu CP (1982) Cytogenetics in domestic animal production. IInd World Congr Genet Appl Livest Prod Madrid, 4-8 octobre, vol 6, 375-384, Editorial Garsi, Madrid. MC : France / bovin / translocation / fertilité / bibliographie

169 Popescu CP (1984) The 1/29 translocation twenty years after. VIth Eur Collog Cytogenet Domest Anim Zurich, 16-21 July, 36-39. MC : France / bovin / translocation 1/29 / fertilité / éradication / bibliographie

170 Popescu CP (1985) Ne Symp Am Cytogénét Anim Domest. Elev Insém 208, 17-18. MC : France / cytogénétique / bovin / porcin / translocation $1 / 29 /$ mise au point

171 Popescu CP (1990) Conséquences des anomalies de la structure chromosomique chez les animaux domestiques. Reprod Nutr Dev (suppl 1) 105s-116s. MC : France / bovin / anomalie chromosomique / chromosome / fertilité

172 Popescu CP, Boscher J (1974) Observations sur une fusion centrique chez les bovins (Bos taurus L). Ist World Congr Genet Appl Livest Prod, Madrid, 7-11 octobre, vol 3, 165168, Editorial Garsi, Madrid. MC : France / bovin / Charolais / Montbeliard / Limousin / Blonde d'Aquitaine / translocation 1/29

173 Popescu CP, Cribiu EP (1977) Étude des anomalies cariotypiques présentées par les reproducteurs. Elev Insém 160, 46-48. MC : France / bovin / Limousin / Pie Noire (Holstein Friesian) / translocation 1/29

174 Popescu CP, Cribiu EP, Poivey JP, Seitz JL (1979) Étude cytogénétique d'une population bovine de Côte-d'lvoire. Rev Elev Méd Vét Pays Trop 32, 81-84. MC : Afrique / Côte d'lvoire / bovin / N'Dama / Baoulé / Gobra / translocation 1/29

175 Popescu CP, Cribiu EP, Tschudi P (1976) Deux cas de fusion centrique chez Bos taurus L en Suisse. Ann Génét Sél Anim 7, 317 319. MC : Suisse / Bovin / Pie Rouge (Simmental) / translocation 1/29

176 Popescu CP, Gauthier D, Tambasco AJ (1987) Etude cytogénétique des bovins créoles élevés en Guadeloupe. Rev Elev Méd Vét Pays Trop 40, 89-91. MC : France / Guadeloupe / créole / Zébu / caryotype / chromosome / cytogénétique

177 Potter WL, Upton PC, Blackshaw AW (1979) Presumptive 1/29 autosomal translocation in Australian cattle. Aust Vet $J$ 55, 209-213. MC : Australie / bovin / Charolais / translocation $1 / 29 /$ fertilité

178 Power R, Masterson J (1973) Chromosome studies in Al Bulls. Ir Vet J 27, 125-128. MC : Irlande / Charolais / bovin / Pie Rouge (Simmental) / Shorthorn / Aberdeen Angus / Hereford / Pie Noire (Frisonne Holstein) / chromosome

179 Queinnec G, Darre R, Berland HM, Raynaud JC (1974) Étude de la translocation 1/29 dans la population bovine du sud-ouest de la France : conséquences zootechniques. Ist World Congr Genet Appl Livest Prod, Madrid, 7-11 octobre, vol 3, 131-151, Editorial Garsi, Madrid. MC : France / bovin / translocation 1/ 29 / Limousin / Blonde d'Aquitaine / fertilité mâle / fertilité femelle / croisement / Charolais / Coopelso 93

180 Rangel-Figueiredo T, lannuzzi L (1990) High incidence of $1 / 29$ translocation in a Portuguese cattle breed. IXth Eur Colloq Cytogenet Domest Anim Toulouse-Auzeville, 7-13 July, 16, abstr. MC : Portugal / bovin / Maronesa / translocation 1/29

181 Refrigeri S, Casciotti D, Martino G, Silvestrelli $M$ (1985) Traslocazione $1 / 29$ in un soggetto Maremmano. (Translocation $1 / 29$ in a Maremma bull). Atti Soc It Sci Vet 39, 193-195. MC : Italie / bovin / translocation 1/29 / Chianina / Maremmana

182 Refsdal AO (1976) Low fertility in daughters of bulls with $1 / 29$ translocation. Acta Vet Scand 17, 190-195. MC : Norvège / bovin / Pie Rouge (Simmental) / fertilité femelle

183 Refsdal AO, Lunaas T, Garm O (1974) Étude de la fertilité des génisses et vaches éliminées à cause de troubles de la reproduction (en norvégien). XII Nord Vet Kongr Reykjavik 1974, Foredrag og rapporter, 272. MC : Norvège / bovin / Pie Rouge (Simmental) / translocation

184 Rieck GW, Hohn H, Herzog A (1968) Familial occurrence of centromere chromosome fusion in cattle (all rés angl). Zuchthyg 3, 
117-182. MC : Allemagne / Pie Rouge (Simmental) / fusion centrique

185 Rugiati S, Fedrigo M (1967) Alteratione cromosomica riscontrata in un toro acondroplasico di della razza Romagnola (ital rés fr angl). Ateneo Parmense - Acta Bio-Medica 38, 3-7. MC : Italie / bovin / Romagnola / translocation

186 Rugiati S, Fedrico M (1968) Anomalie cromosomiche riscontrate in bovini controdistrofici razza Romagnolia (ital rés fr angl). Ateneo Parmense 39, 457-470. MC : Italie / bovin / Romagnola / translocation

187 Ruliang Y, Lin CC, Danbing W, Youchun C, Entang $J$ (1986) (Preliminary study of $1 / 29$ chromosome translocation of Simmental cattle in inner Mongolia) (en chinois, abstr). Acta Vet Zootech Sinica 17, 1-6. MC : Chine / bovin / translocation 1/29 / Pie Rouge (Simmental)

188 Samarineanu M (1979) Cercetari citogenetice in relatie cu functia de reproductie la taurine (en roumain). Rev Crest Anim 1, 15-18. MC : Roumanie / bovin / chimérisme / translocation $1 / 29$ / fertilité

189 Samarineanu M, Livescu BE, Granciu I (1977) (L'identification de la translocation autosomale Roberstonienne chez quelques races des bovins de Roumanie) (roum rés angl fr all rus). Lucr Stiint Inst Cercet Crest Taurinelor-Corbeanca 3, 53-60. MC : Roumanie / bovin / Pie Noire (Holstein Frisonne) / Brune des Alpes (Brown Swiss) / Charolais / translocation

190 Schifferli RC (1981) (A study of karyotypes of bulls at an Al centre) (jap rés angl). Arch Med Vet 13, 29-38. MC : Argentine / Pie Noire (Holstein Frisonne) / Pie Rouge (Simmental) / translocation 1/29

191 Schmutz SM, Moker J (1989) Impact of a 1/ 29 Robertsonian translocation on a herd of purebred beef cattle. Can J Anim Sci 69, 891-896. MC : Canada / bovin / Charolais / translocation $1 / 29$

192 Schmutz SM, Moker J (1989) Incidence of the 1/29 Robertsonian translocation in Western Canadian beef cattle. VIth N Am Colloq Cytogenet Domest Anim, Purdue Univ, July, 10. MC : Canada / bovin / Charolais / translocation $1 / 29$

193 Sellyei M, Kovacs A, Vass L, Meszaros I (1975) (Studies on the chromosomes of Al bulls) (hongr rés angl fr rus). Magy Allator Lap 30, 699-706. MC : Hongrie / bovin / fertilité / translocation

194 Shinzato G, Shinio A, Tokashiki S (1980) (Centric fusion of chromosomes from Japanese Black cattle) (jap rés angl). Sci Bull Coll Agric Ryukyus Univ (27), 285-292. MC : Japon / Noire Japonaise (Japanese Black) I translocation 1/29

195 Silvestrelli M, Casciotti D, Valfre F, Chicchini U (1981) Robertsonian translocation in a Chianina cow and in its offspring. Ann Génét Sél Anim 13, 429-434. MC : Italie / bovin / Chianina / translocation 1/29 / fertilité

196 Sitko M, Dianovsky J (1981) (Genetic analysis in commercial herds of cattle in Slovakia) (en tchèque). Veterinarstvi 31, 155-157. MC : Tchécoslovaquie / bovin / translocation 1/29 / pathologie

197 Slanina L, Konrad V, Vajda V, Lojda L, Zibrin $M$ et al (1978) (Congenital neurofibromatosis of the skin in calves) (all rés angl). Dtsch Tierarztl Wochenschr 85, 41-54. MC : Tchécoslovaquie / bovin / Pie Rouge (Simmental) / anomalie chromosomique / translocation 1/29

198 Slanina L, Konrad V, Vajda V et al (1976) (Mass outbreak of congenital cutaneous neurofibromatosis in calves) (en tchèque). Veterinarstvi 26, 245-249. MC : Tchécoslovaquie / bovin / Pie Rouge (Simmental) / translocation $1 / 29$

199 Slota E, Danielak B (1984) (A case of $1 / 29$ Robertsonian translocation in a Black and White cow) (pol rés angl rus all). Roczn Nauk Zootech 11, 9-14. MC : Pologne / bovin / Pie Noire (Holstein Frisonne) / translocation 1/29

200 Slota E, Danielak B, Kozubska-Sobocinska A (1988) The Robertsonian translocation in the cattle quintuplet. VIIIth Eur Colloq Cytogenet Domest Anim, Bristol, 19-22 July, 122-124. MC : Pologne / bovin / Pie Rouge (Simmental) / translocation 1/29

201 Smirnov OK (1989) (Les anomalies cytogénétiques des chromosomes du bétail Simmental) (russe). Zootekhnija (7), 17-19. MC : URSS / bovin / Pie Rouge (Simmental) / translocation $1 / 29$

202 Smirnov OK, Goldman IL, Konovalov MA, llyushina IA (1988) (Les anomalies chromosomiques des taureaux de race Simmental) 
(en russe). Zooteckhnija (10), 24-25. MC : URSS / bovin / Pie Rouge (Simmental) / anomalie chromosomique

203 Soldatovic B, Prolic Z, Cvetkovic N (1977) (The significance of cytogenetics in the diagnosis of congenital anomalies in cattle) (yougos rés angl). J Veterinorski Glasnik 31, 33-40. MC : Yougoslavie / bovin / Pie Rouge (Simmental) / translocation / anomalie chromosomique / fertilité femelle

204 Stanik J (1985) (The use of cytogenetic tests for recording of genetic health and in genetic selection of livestock) (roumain). Veterinarstvi 35 (4), 162-164. MC : Roumanie / bovin / ovin / porcin / anomalie chromosomique

205 Stranzinger G (1976) Chromosomenuntersuchung als zuchthygienische Massnahme bei unseren Zuchttieren (en allemand). Tierzuchter 2, 67-68. MC : Suisse / bovin / translocation 1/29 / bibliographie

206 Stranzinger G, Dolf G, Fries R, Stocker $H$ (1981) Some rare cases of chimerism in twin cattle and their proposed use in determining germinal cell migration. J Hered 72, 360-362. MC : Suisse / bovin / Aberdeen Angus / Pie Rouge (Simmental) / Brune des Alpes (Brown Swiss) / translocation 1/29 / chimérisme

207 Stranzinger G, Dolf G, Marki U, Hunziker R, Stampfli G (1986) Zytogenetische und biochemische Untersuchungen bei der Erzeugung von Injektionschimaren beim rind (Cytogenetic and biochemical investigations to produce an injection chimaera in cattle). Zuchthyg 21, 1-7. MC : Suisse / bovin / translocation 1/29 / chimérisme

208 Stranzinger G, Emler K, Bauer G, Gaillard C (1981) (Conception datas from the experiment with mixed semen of bulls differing in breed and chromosome number) (all rés angl). Zuchthyg 16, 49-53. MC : Suisse / bovin / Brune des Alpes (Brown Swiss) / Pie Rouge (Simmental) / Aberdeen Angus / translocation $1 / 29$ / fertilité

209 Stranzinger G, Forster M (1976) (Autosomal chromosome translocation of Piebald cattle and Brown cattle) (all rés angl). Experientia 32, 24-27. MC : Allemagne / bovin / translocation / Brune des Alpes (Brown Swiss) / Piebald / fertilité

210 Succi G, Giovanni AM de (1976) (Sur la présence de la translocation 1/29 dans des races bovines italiennes) (ital rés angl fr). Atti Soc it Sci Vet 30, 486-489. MC : Italie / bovin / Chianina / Marchigiana / Modenese / translocation $1 / 29$

211 Succi G, Giovanni AM de, Molteni L (1976) Nouvelles observations sur une translocation robertsonienne en race bovine Romagnole. Ann Génét Sél Anim 8, 37-40. MC : Italie / bovin / Romagnola / translocation 1/29

212 Succi G, Molteni L, Giovanni AM de (1979) (First observations on the fertility of daughters of 1/29 translocation carrier bulls) (ital rés angl). Atti Conv Naz Soc it Sci Vet 33, 215. MC : Italie / bovin / translocation 1/29 / fertilite

213 Succi G, Molteni L, Giovanni AM de (1980) Cytogenetic study of some Italian cattle breeds on the decrease or in the process of extinction. IVth Eur Collog Cytogenet Domest Anim, Uppsala, 10/13 juin, 136-140. MC : Italie / bovin / Romagnola / Chianina / Marchigiana / Modicana / Ottonese / Grise Alpine / translocation / fertilité femelle

214 Swartz HA, Vogt DW (1983) Chromosome abnormalities as a cause of reproductive inefficiency in heifers. $J$ Hered 74, 320-324. MC : Etats-Unis / bovin / anomalie chromosomique / Rouge sans cornes (Red Poll) / Marc II / Pie Rouge (Simmental) / Pinzgauer / Limousin / Hereford / Aberdeen Angus / Charolais / mosaïque / chimérisme / translocation 1/29

215 Swensson T (1973) Hereditary chromosome translocation causing lowered fertility in a cattle population. Measures for assessing and eliminating carriers. XXIVth Annu Meet Eur Assoc Anim Prod Anim Prod, Vienna, Austria, September 23-26. MC : Suéde / bovin / Pie Rouge (Simmental) / translocation / fertilité femelle

216 Switonski M, Gustavsson I, Ansari HA / Stranzinger $\mathrm{G}$ (1988) Centric fusion translocation behaviour in the first meiotic prophase, studied by synaptonemal complex analysis in cattle, goat and blue fox. VIIIth Eur Collog Cytogenet Domest Anim Bristol, 19-22 July, 66-70. MC : Suède / bovin / translocation 1/29

217 Sysa PS (1976) (Chromosomal translocation $1 / 29$ in two young bulls of Polish Red breed) (pol rés angl). Med Weter 32, 353-355. MC : Pologne / bovin / Pie Rouge (Simmental) / Pie Noire (Holstein Frisonne) $\times$ Pie Rouge (Simmental) / fertilité / translocation 1/29 
218 Tschudi P (1984) Twelve years of cytogenetic investigation in Al bulls in Switzerland. Vith Eur Colloq Cytogenet Domest Anim, Zurich, Suisse, July 16-20, 40-42. MC : Suisse / bovin / cytogénétique / Pie Rouge (Simmental) / Brune des Alpes (Brown Swiss) / Freiburg / Erigner / translocation 1/29

219 Tschudi P, Zahner B, Kupfer U, Stampfli G (1977) Chromosomenuntersuchungen an schweiizerischen Rinderrassen (all rés angl ital). Schweiz Arch Tierheilk 119, 329-336. MC : Suisse / bovin / Pie Rouge (Simmental) / Brune des Alpes (Brown Swiss) / anomalie chromosomique / translocation robertsonienne

220 Weber AF, Buoen LC, Terhaar BL, Ruth GR, Momont HW (1989) Low fertility related to 1/ 29 centric fusion anomaly in cattle. $J \mathrm{Am}$ Vet Med Assoc 195, 643-646. MC : États-Unis / bovin / Pie Rouge (Simmental) / translocation $1 / 29$

221 Wilson TD (1990) Identification of the $1 / 29$ Robertsonian translocation chromosome in British Friesian cattle. Vet Rec 126 (2), 3739. MC : Grande-Bretagne / bovin / Pie Noire (Holstein Frisonne) / translocation 1/29

222 Zahner B, Kupfer U, Tschudi P (1979) (The influence of the 1/29 translocation on fertility of Simmental cows in Switzerland) (all rés angl). Zuchthyg 14, 49-54. MC : Suisse / bovin / Pie Rouge (Simmental) / translocation $1 / 29$ / fertilité femelle

223 Zarazaga I, Arruga MV (1982) A Robertsonian translocation in a bull-fighting (de lidia) bull. Arch Zootec 31, 91-95. MC : Espagne / bovin / taureau de combat / translocation 1/29

224 Zarazaga I, Arruga MV (1982) Cytogenetic studies in Spanish cattle breeds. Vth Eur Colloq Cytogenet Domest Anim, Proc, MilanoGargnano, June 7-11, 62-70 Milan, Italie. MC : Espagne / bovin / taureau de combat

225 Zhigachev AE, Nikitin NS, Cherkasov VV (1984) (The frequency of the $1 / 29$ chromosome translocation in a randomly selected population of cattle). DokJ Vses Akad S'Kh Nauk 4, 21-22. MC : URSS / bovin / translocation 1/29

226 Zhigachev Al (1984) (The use of cytogenetics in selection and veterinary care). Veteri- nariya 12, 45-57. MC : URSS / bovin / cytogénétique / Tachetée de l'Est / Pie Noire (Holstein Frisonne) / translocation 1/29

227 Zhigachev Al (1986) (Reasons for the 1/29 translocation occurrence in Pale-Motley cattle and its race occurrence in Black and White cattle) (en russe). Dokl Vses Ord Lenina Ord Trud Krasn Znam Akad S'Kh Nauk Im Lenina 9, 30-31. MC : URSS / bovin / translocation 1/29 / Paille Tachetée / Pie Noire (Holstein Frisonne)

228 Zhigachev Al (1986) (The 1/29 chromosome translocation in cattle: its dissemination the mechanism of its formation and its effect on fertility). S'Kh Biol 5, 13-23. MC : URSS / bovin / translocation 1/29 / reproduction / cytologie

229 Zhigachev Al (1989) (Le contrôle cytogénétjque des taureaux) (en russe). Zootekhnija 6, 24-26. MC : URSS / bovin / races européennes / translocation $1 / 29$

230 Zhigachev Al, Nikitin NS, Cherkasov VV, Kurchanov NA, Marusyak VG / Konovalova NN / Grigoryeva NV, Cheredeeva MN (1984) Cytogenetic analysis in populations of cattle. I. Frequency of translocations in an individual population of dairy and beef cattle. Genetika 20 (8), 1360-1364. MC : URSS / bovin / Pie Noire (Holstein Frisonne) / Holmogorskaya / Sychevka / translocation 1/29 / fertilité

231 Zwiauer D, Menken M, Stranzinger $G$, Dempfle L (1980) Auswirkung einer Zentromerfusion auf Form- und Leistungsmerkmale beim rind (all rés angl). Zuchthyg 15, 97-102. MC : Suisse / bovin / translocation 1/29 / fertilité femelle / production laitière / mensurations corporelles

\section{BIBLIOGRAPHIE}

Bougler J (1985) Inventaire des ressources génétiques animales françaises. Tome $\|$ : Bovins. INA Paris

Mason IL (1988) A world dictionary of livestock breeds, types and varieties. $\mathrm{CAB}$ international, Wallingford, Oxon 


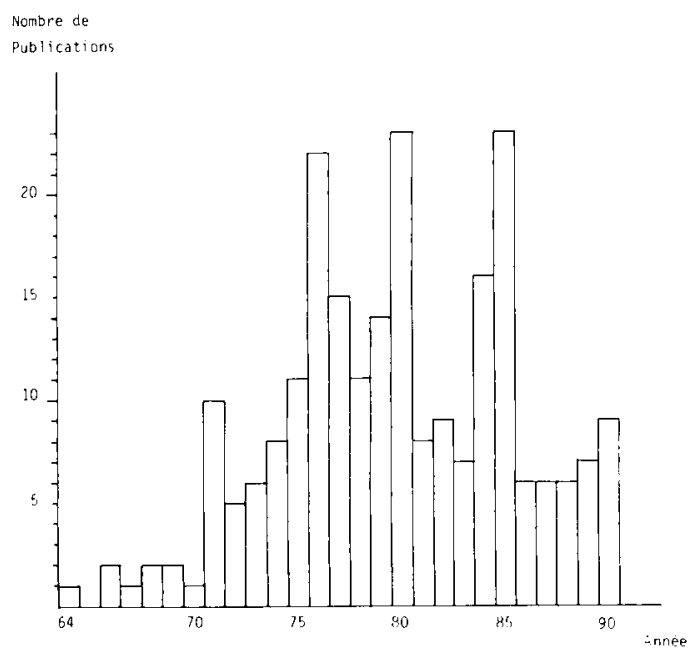

Fig 1. Évolution quantitative des publications par année.

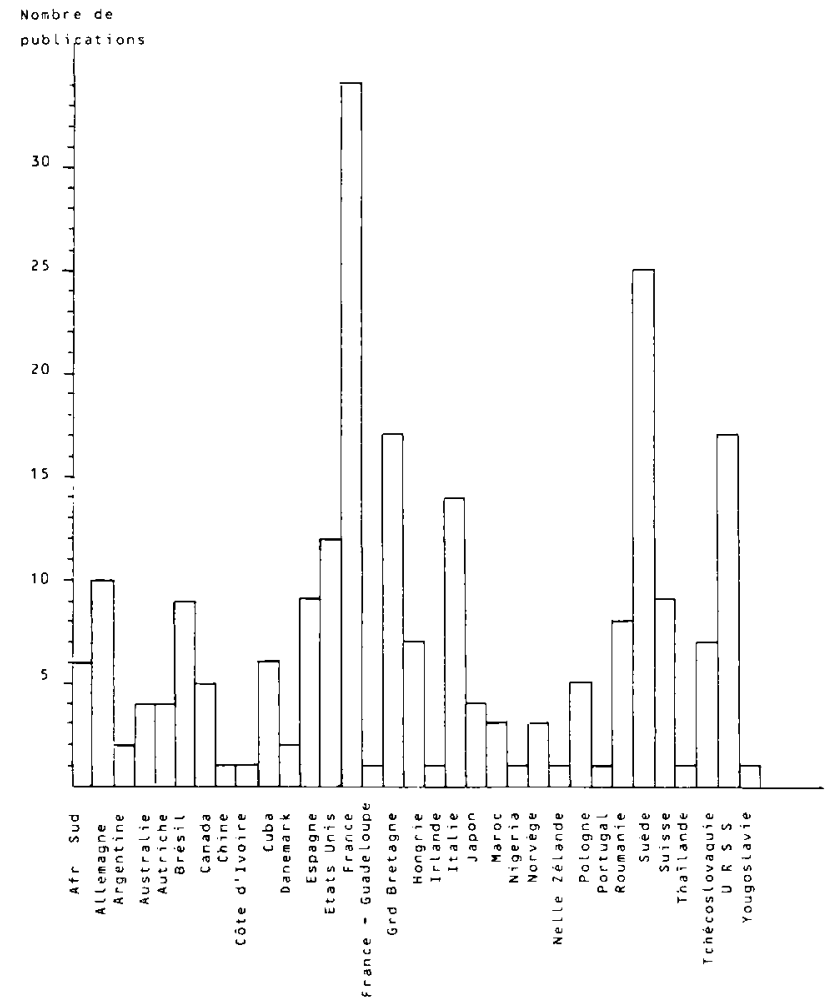

Fig 2. Nombre de publications par pays. 


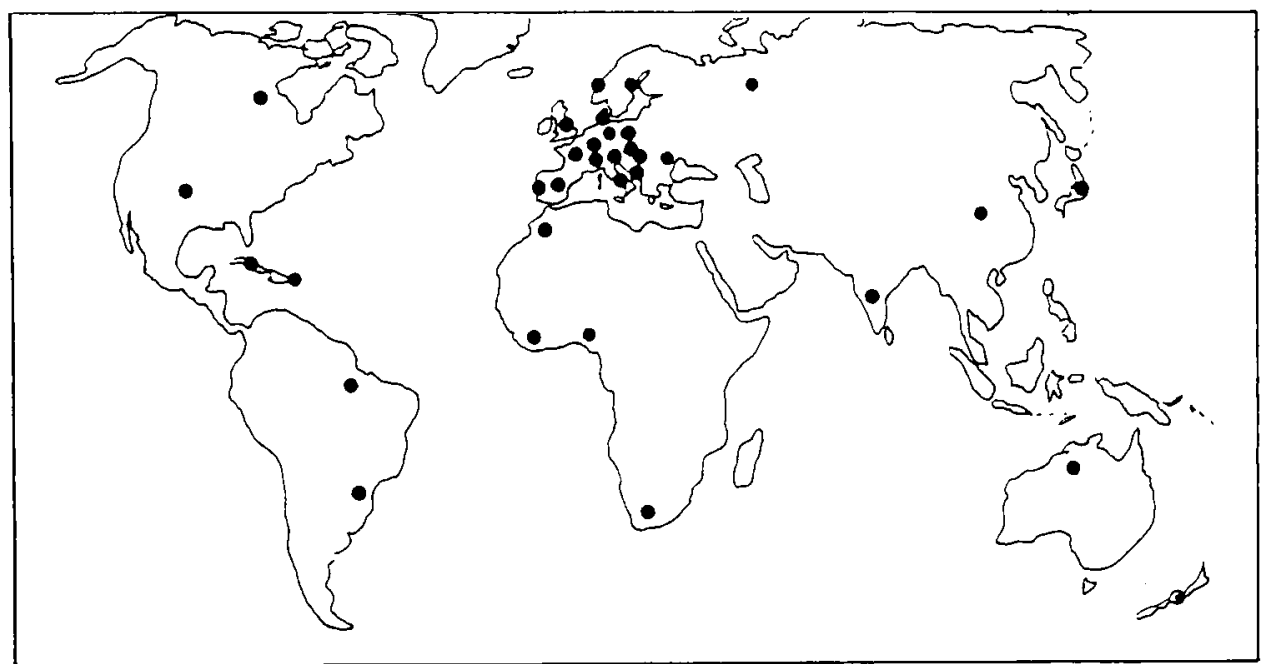

Fig 3. Distribution géographique. Afrique du Sud, Allemagne, Argentine, Australie, Autriche, Brésil, Canada, Chine, Côte d'lvoire, Cuba, Danemark, Espagne, États-Unis, France, France-Guadeloupe, Grande-Bretagne, Hongrie, Italie, Japon, Maroc, Nigeria, Norvège, Nouvelle Zélande, Pologne, Portugal, Roumanie, Suède, Suisse, Thaillande, Tchécoslovaquie, URSS, Yougoslavie. 
Tableau I. Fréquences des porteurs hétérozygotes par race.

\begin{tabular}{|c|c|c|c|c|c|}
\hline Races & \multicolumn{2}{|c|}{ Fréquence (\%) } & Cas signalés & Pays & Ref \\
\hline 15 races & & & 4 & États-Unis & 038 \\
\hline Baoulé & 4,69 & (3 sur 64$)$ & & Côte d'Ivoire & 028 \\
\hline Baoulé & & & 5 & Côte d'lvoire & 174 \\
\hline Blanche Britannique (Bristish White) & 78,85 & (41 sur 52$)$ & & Grande-Bretagne & 036 \\
\hline Blanche Britannique (Bristish White) & 70,40 & & & Grande-Bretagne & 122 \\
\hline Blonde d'Aquitaine & 33,70 & (sur 228) & & Danemark & 084 \\
\hline Blonde d'Aquitaine & 14,24 & (302 sur 2121 ) & & France & 053 \\
\hline Blonde d'Aquitaine & & & 3 & France & 166 \\
\hline Blonde d'Aquitaine & 10,71 & & & France & 172 \\
\hline Blonde d'Aquitaine & 19,61 & $(10$ sur 51$)$ & & France & 049 \\
\hline Blonde d'Aquitaine & 20,00 & (45 sur 225 ) & & France & 179 \\
\hline Blonde d'Aquitaine & & & 1 & Nouvelle-Zélande & 020 \\
\hline Brahman & 0,53 & (1 sur 190$)$ & & Afrique du Sud & 143 \\
\hline Brune de l'Atlas & 25,00 & $(1$ sur 4$)$ & & Maroc & 045 \\
\hline Brune de l'Atlas & 8,26 & (9 sur 109 ) & & Maroc & 123 \\
\hline Brune des Alpes (Brown Swiss) & & & 1 & Allemagne & 209 \\
\hline Brune des Alpes (Brown Swiss) & 10,37 & (31 sur 299) & & États-Unis & 017 \\
\hline Brune des Alpes (Brown Swiss) & 7,14 & (1 sur 14$)$ & & États-Unis & 039 \\
\hline Brune des Alpes (Brown Swiss) & 6,44 & (17 sur 264$)$ & & Etats-Unis & 038 \\
\hline Brune des Alpes (Brown Swiss) & & & 1 & Italie & 060 \\
\hline Brune des Alpes (Brown Swiss) & 0,40 & (4 sur 998 ) & & Suisse & 218 \\
\hline Brune des Alpes (Brown Swiss) & & & 2 & Tchécoslovaquie & 010 \\
\hline Cachena & 3,70 & & & Espagne & 003 \\
\hline Charolais & 2,47 & (2 sur 81$)$ & & Brésil & 135 \\
\hline Charolais & 45,74 & (43 sur 94 ) & & Canada & 192 \\
\hline Charolais & 30,95 & (13 sur 42$)$ & & Etats-Unis & 021 \\
\hline Charolais & & & 1 & France & 054 \\
\hline Charolais & 3,28 & $(12$ sur 366$)$ & & France & 049 \\
\hline Charolais & 4,76 & & & France & 172 \\
\hline Charolais & & & 1 & Grande-Bretagne & 087 \\
\hline Charolais & 0,54 & (1 sur 185$)$ & & Grande-Bretagne & 091 \\
\hline Charolais & & & 2 & Grande-Bretagne & 116 \\
\hline Charolais & & & 1 & Grande-Bretagne & 030 \\
\hline Charolais & 30,77 & ( 4 sur 13 ) & & Pologne & 100 \\
\hline Charolais & & & 1 & Roumanie & 113 \\
\hline Charolais & & & 1 & Roumanie & 189 \\
\hline Chianina & 1,39 & (1 sur 72 ) & & Brésil & 043 \\
\hline Chianina & & & 1 & Italie & 195 \\
\hline Chianina & & & 1 & Italie & 210 \\
\hline Chianina & 9,52 & (2 sur 21 ) & & Italie & 213 \\
\hline Coopelso 93 & 28,57 & ( 8 sur 28 ) & & France & 179 \\
\hline Créole & & & 1 & France-Guadeloupe & 176 \\
\hline Grise Autrichienne (Tyrol Grey) & 20,00 & $(1$ sur 5$)$ & & Autriche & 130 \\
\hline Grise des Steppes & 3,77 & (4 sur 106$)$ & & Hongrie & 108 \\
\hline Grise des Steppes & & & 1 & Hongrie & 110 \\
\hline Grise des Steppes & 9,09 & ( 7 sur 77$)$ & & Hongrie & 109 \\
\hline Guernsey & 15,79 & (3 sur 19) & & Canada & 018 \\
\hline Holmogorskaya & 10,00 & & & URSS & 230 \\
\hline Lebedin & 7,64 & (63 sur 825$)$ & & URSS & 101 \\
\hline
\end{tabular}


Tableau I (suite)

\begin{tabular}{|c|c|c|c|c|c|}
\hline Races & \multicolumn{2}{|c|}{ Fréquence (\%) } & Cas signalé & Pays & Ref \\
\hline Lebedin & 6,72 & (42 sur 625 ) & & URSS & 102 \\
\hline Lidia & 6,25 & & & Espagne & 003 \\
\hline Lidia & 5,08 & ( 3 sur 59 ) & & Espagne & 224 \\
\hline Limousin & 5,84 & ( 8 sur 137 ) & & France & 049 \\
\hline Limousin & 0,41 & (2 sur 492 ) & & France & 173 \\
\hline Limousin & 5,63 & (13 sur 231) & & France & 179 \\
\hline Limousin & 8,21 & & & France & 172 \\
\hline Marc II & & & 1 & Etats-Unis & 214 \\
\hline Marchigiana & 36,36 & (4 sur 11) & & Brésil & 152 \\
\hline Marchigiana & 18,75 & ( 3 sur 16$)$ & & Italie & 213 \\
\hline Marchigiana & & & 1 & Italie & 210 \\
\hline Maremmana & & & 1 & Italie & 181 \\
\hline Maronesa & 49,54 & (54 sur 109 ) & & Portugal & 180 \\
\hline Modicana & & & 1 & Italie & 056 \\
\hline Modicana & 6,45 & (2 sur 31 ) & & Italie & 213 \\
\hline Montbéliard & 0,86 & (2 sur 232) & & France & 027 \\
\hline Montbéliard & & & 2 & France & 155 \\
\hline Montbéliard & 0,27 & (4 sur 1463 ) & & France & 162 \\
\hline Montbéliard & 3,50 & & & France & 172 \\
\hline Montbéliard & & & 2 & URSS & 140 \\
\hline Montbéliard & 7,64 & (63 sur 825$)$ & & URSS & 101 \\
\hline Montbéliard & 6,72 & (42 sur 625$)$ & & URSS & 102 \\
\hline Morucha & 17,60 & & & Espagne & 003 \\
\hline Nguni & 10,16 & (31 sur 305) & & Afrique du Sud & 142 \\
\hline Noire Japonaise (Japanese Black) & 16,07 & $(18$ sur 112$)$ & & Japon & 082 \\
\hline Noire Japonaise (Japanese Black) & 12,73 & $(7$ sur 55$)$ & & Japon & 125 \\
\hline Noire Japonaise (Japanese Black) & & & 1 & Japon & 194 \\
\hline Non indiqué & & & 1 & Espagne & 002 \\
\hline Non indiqué & & & 3 & États-Unis & 037 \\
\hline Non indiqué & & & 3 & France & 052 \\
\hline Non indiqué & 18,18 & (2 sur 11$)$ & & Hongrie & 193 \\
\hline Non indiqué & & & 1 & Tchécoslovaquie & 196 \\
\hline Non indiqué & & & 1 & URSS & 059 \\
\hline Non indiqué & 17,02 & ( 8 sur 47$)$ & & URSS & 225 \\
\hline Ottonese & 3,57 & (1 sur 28$)$ & & Italie & 213 \\
\hline Paille tachetée & 0,16 & (44 sur 281 ) & & URSS & 227 \\
\hline Pedi & 20,00 & $(1$ sur 5$)$ & & Atrique du Sud & 142 \\
\hline Pie Noire (Holstein Friesian) & 0,41 & (2 sur 492 ) & & France & 173 \\
\hline Pie Noire (Holstein Friesian) & & & 1 & Espagne & 006 \\
\hline Pie Noire (Holstein Friesian) & & & 1 & Etats-Unis & 092 \\
\hline Pie Noire (Holstein Friesian) & 0,30 & (1 sur 330$)$ & & Grande-Bretagne & 154 \\
\hline Pie Noire (Holstein Friesian) & 9,52 & (6 sur 63$)$ & & Hongrie & 138 \\
\hline Pie Noire (Holstein Friesian) & & & 1 & Pologne & 199 \\
\hline Pie Noire (Holstein Friesian) & & & 1 & URSS & 008 \\
\hline Pie Noire (Holstein Friesian) & & & 1 & URSS & 058 \\
\hline Pie Noire (Holstein Friesian) & 20,00 & & & URSS & 226 \\
\hline Pie Noire (Holstein Friesian) & 0,20 & (1 sur 506$)$ & & URSS & 227 \\
\hline Pie Noire (Holstein Friesian) & 10,00 & & & URSS & 230 \\
\hline Pie Noire (Holstein Friesian) & 7,64 & (63 sur 825$)$ & & URSS & 101 \\
\hline Pie Rouge (Simmental) & 3,57 & (1 sur 28$)$ & & Allemagne & 093 \\
\hline
\end{tabular}


Tableau I (suite)

\begin{tabular}{|c|c|c|c|c|c|}
\hline Races & \multicolumn{2}{|c|}{ Fréquence (\%) } & Cas signalé & Pays & Ref \\
\hline Pie Rouge (Simmental) & & & 2 & Allemagne & 095 \\
\hline Pie Rouge (Simmental) & & & 1 & Allemagne & 184 \\
\hline Pie Rouge (Simmental) & 4,35 & (1 sur 23 ) & & Argentine & 190 \\
\hline Pie Rouge (Simmental) & 2,11 & ( 3 sur 142$)$ & & Autriche & 131 \\
\hline Pie Rouge (Simmental) & 1,07 & (3 sur 280$)$ & & Autriche & 132 \\
\hline Pie Rouge (Simmental) & & & 4 & Chine & 187 \\
\hline Pie Rouge (Simmental) & & & 2 & États-Unis & 214 \\
\hline Pie Rouge (Simmental) & & & 1 & Grande-Bretagne & 030 \\
\hline Pie Rouge (Simmental) & 7,69 & (2 sur 26$)$ & & Grande-Bretagne & 089 \\
\hline Pie Rouge (Simmental) & 2,65 & (3 sur 113 ) & & Grande-Bretagne & 091 \\
\hline Pie Rouge (Simmental) & 2,88 & (16 sur 556$)$ & & Hongrie & 109 \\
\hline Pie Rouge (Simmental) & 2,63 & (2 sur 76$)$ & & Hongrie & 146 \\
\hline Pie Rouge (Simmental) & 25,00 & (1 sur 4$)$ & & Hongrie & 146 \\
\hline Pie Rouge (Simmental) & 4,18 & $(18$ sur 430$)$ & & Norvège & 001 \\
\hline Pie Rouge (Simmental) & 17,14 & (24 sur 140$)$ & & Norvège & 183 \\
\hline Pie Rouge (Simmental) & & & 1 & Nouvelle-Zélande & 020 \\
\hline Pie Rouge (Simmental) & & & 5 & Pologne & 200 \\
\hline Pie Rouge (Simmental) & & & 1 & Pologne & 217 \\
\hline Pie Rouge (Simmental) & 1,50 & (3 sur 200 ) & & Roumanie & 024 \\
\hline Pie Rouge (Simmental) & & & 1 cas $1 / 29$ & Roumanie & 025 \\
\hline Pie Rouge (Simmental) & 21,05 & (12 sur 57$)$ & & Suède & 035 \\
\hline Pie Rouge (Simmental) & 7,14 & (2 sur 28$)$ & & Suède & 0,61 \\
\hline Pie Rouge (Simmental) & 14,33 & (293 sur 2045 ) & & Suède & 063 \\
\hline Pie Rouge (Simmental) & 14,00 & & & Suède & 064 \\
\hline Pie Rouge (Simmental) & 12,50 & (118 sur 944$)$ & & Suède & 067 \\
\hline Pie Rouge (Simmental) & & & 3 & Suède & 078 \\
\hline Pie Rouge (Simmental) & 14,50 & (sur 2000 ) & & Suède & 215 \\
\hline Pie Rouge (Simmental) & 1,84 & (23 sur 1251 ) & & Suisse & 218 \\
\hline Pie Rouge (Simmental) & 9,52 & (4 sur 42$)$ & & Tchécoslovaquie & 119 \\
\hline Pie Rouge (Simmental) & 5,70 & & & Tchécoslovaquie & 197 \\
\hline Pie Rouge (Simmental) & & & 1 & Tchécoslovaquie & 198 \\
\hline Pie Rouge (Simmental) & 7,64 & (63 sur 825 ) & & URSS & 101 \\
\hline Pie Rouge (Simmental) & 6,72 & (42 sur 625 ) & & URSS & 102 \\
\hline Pie Rouge (Simmental) & & & 3 & URSS & 201 \\
\hline Pie Rouge (Simmental) & 8,11 & (3 sur 37 ) & & URSS & 202 \\
\hline Pie Rouge (Simmental) & 1,49 & (1 sur 67 ) & & Yougoslavie & 203 \\
\hline Piebald & & & 1 & Allemagne & 209 \\
\hline Pitangueiras & 50,00 & ( 3 sur 6 ) & & Brésil & 136 \\
\hline Pitangueiras & 25,00 & ( 3 sur 12$)$ & & Brésil & 149 \\
\hline Pitangueiras & 27,00 & & & Brésil & 150 \\
\hline Pitangueiras & 27,50 & (66 sur 240$)$ & & Brésil & 151 \\
\hline Podolian & 32,20 & (19 sur 59$)$ & & Italie & 009 \\
\hline Race Corse & 28,57 & (24 sur 84 ) & & France & 085 \\
\hline Races Bavaroises & 0,30 & ( 4 sur 1342$)$ & & Allemagne & 047 \\
\hline Races Espagnoles & 20,28 & (29 sur 143$)$ & & Espagne & 007 \\
\hline Races européennes & 6,84 & (54 sur 789 ) & & URSS & 229 \\
\hline Red Bororo & 10,00 & $(1$ sur 10$)$ & & Nigeria & 147 \\
\hline Retinta & 27,78 & $(5$ sur 18$)$ & & Espagne & 137 \\
\hline Retinta & 25,30 & & & Espagne & 003 \\
\hline
\end{tabular}


Tableau I (suite)

\begin{tabular}{|c|c|c|c|c|}
\hline Races & Fréquence (\%) & Cas signalé & Pays & Ref \\
\hline Romagnola & 22,07 & & Italie & 040 \\
\hline Romagnola & 28,57 & & Italie & 055 \\
\hline Romagnola & (2 sur 4$)$ & & Italie & 186 \\
\hline Romagnola & $(2 \operatorname{sur} 9)$ & & Italie & 211 \\
\hline Romagnola & $22,27 \quad(49$ sur 220$)$ & & Italie & 213 \\
\hline Rouge sans cornes (Red Poll) & & 2 & Australie & 081 \\
\hline Rouge sans cornes (Red Poll) & & 2 & Etats-Unis & 214 \\
\hline Santa Gertrudis & & 2 & Cuba & 011 \\
\hline Santa Gertrudis & ( 3 sur 10$)$ & & Cuba & 016 \\
\hline Santa Gertrudis & & 3 & Cuba & 016 \\
\hline Sayaguesa & 24,00 & & Espagne & 003 \\
\hline Sychevka & 10,00 & & URSS & 230 \\
\hline Taureau de Combat & & 3 & Espagne & 004 \\
\hline Taureau de Combat & & 1 & Espagne & 223 \\
\hline Thai & & 2 & Thailande & 044 \\
\hline Zebu Malawi & 1,90 & & Afrique du Sud & 142 \\
\hline
\end{tabular}


Tableau II. Fréquences des porteurs hétérozygotes par pays.

\begin{tabular}{|c|c|c|c|c|c|}
\hline Pays & Races & Fré & quence (\%) $c$ & Cas signalé & Ref \\
\hline Afrique du Sud & $\begin{array}{c}\text { Nguni } \\
\text { Pedi } \\
\text { Zebu Malawi }\end{array}$ & $\begin{array}{r}10,16 \\
20,00 \\
1,90\end{array}$ & $\begin{array}{l}(31 \text { sur } 305) \\
(1 \text { sur } 5)\end{array}$ & 1 & 142 \\
\hline Afrique du Sud & Brahman & 0,53 & $(1$ sur 190$)$ & & 143 \\
\hline Allemagne & Races Bavaroises & 0,30 & (4 sur 1342$)$ & & 047 \\
\hline Allemagne & Pie Rouge (Simmental) & 3,57 & (1 sur 28) & & 093 \\
\hline Allemagne & Pie Rouge (Simmental) & & & 2 & 095 \\
\hline Allemagne & Pie Rouge (Simmental) & & & 1 & 184 \\
\hline Allemagne & $\begin{array}{c}\text { Brune des Alpes (Brown Swiss) } \\
\text { Piebald }\end{array}$ & & & $\begin{array}{l}1 \\
1\end{array}$ & 209 \\
\hline Argentine & Pie Rouge (Simmental) & 4,35 & (1 sur 23) & & 190 \\
\hline Australie & Rouge sans cornes (Red Poll) & & & 2 & 081 \\
\hline Autriche & Grise Autrichienne (Tyrol Grey) & 20,00 & (1 sur 5$)$ & & 130 \\
\hline Autriche & Pie Rouge (Simmental) & 2,11 & ( 3 sur 142$)$ & & 131 \\
\hline Autriche & Pie Rouge (Simmental) & 1,07 & (3 sur 280 ) & & 132 \\
\hline Brésil & Chianina & 1,39 & (1 sur 72$)$ & & 043 \\
\hline Brésil & Charolais & 2,47 & (2 sur 81 ) & & 135 \\
\hline Brésil & Pitangueiras & 50,00 & ( 3 sur 6$)$ & & 136 \\
\hline Brésil & Pitangueiras & 25,00 & ( 3 sur 12$)$ & & 149 \\
\hline Brésil & Pitangueiras & 27,00 & & & 150 \\
\hline Brésil & Pitangueiras & 27,50 & (66 sur 240$)$ & & 151 \\
\hline Brésil & Marchigiana & 36,36 & $(4 \operatorname{sur} 11)$ & & 152 \\
\hline Canada & Guernsey & 15,79 & (3 sur 19) & & 018 \\
\hline Canada & Charolais & 45,74 & (43 sur 94 ) & & 192 \\
\hline Chine & Pie Rouge (Simmental) & & & 4 & 187 \\
\hline Côte d'lvoire & Baoulé & 4,69 & (3 sur 64$)$ & & 028 \\
\hline Côte d'ivoire & Baoulé & & & 5 & 174 \\
\hline Cuba & Santa Gertrudis & & & 2 & 011 \\
\hline Cuba & Santa Gertrudis & 30,00 & ( 3 sur 10$)$ & & 015 \\
\hline Cuba & Santa Gertrudis & & & 3 & 016 \\
\hline Danemark & Blonde d'Aquitaine & 33,70 & (sur 228) & & 084 \\
\hline Espagne & Non indiqué & & & 1 & 002 \\
\hline \multirow[t]{5}{*}{ Espagne } & Lidia & 6,25 & & & 003 \\
\hline & Sayaguesa & 24,00 & & & \\
\hline & Cachena & 3,70 & & & \\
\hline & Morucha & 17,60 & & & \\
\hline & Retinta & 25,30 & & & \\
\hline Espagne & Taureau de Combat & & & 3 & 004 \\
\hline Espagne & Pie Noire (Holstein Friesian) & & & 1 & 006 \\
\hline Espagne & Races Espagnoles & 20,28 & (29 sur 143 ) & & 007 \\
\hline Espagne & Retinta & 27,78 & (5 sur 18$)$ & & 137 \\
\hline Espagne & Taureau de Combat & & & 1 & 223 \\
\hline Espagne & Lidia & 5,08 & ( 3 sur 59 ) & & 224 \\
\hline États-Unis & Brune des Alpes (Brown Swiss) & 10,37 & (31 sur 299) & & 017 \\
\hline États-Unis & Charolais & 30,95 & (13 sur 42$)$ & & 021 \\
\hline Etats-Unis & Non indiqué & & & 3 & 037 \\
\hline \multirow[t]{2}{*}{ Etats-Unis } & 15 races & & & 4 & 038 \\
\hline & Brune des Alpes (Brown Swiss) & 6,44 & (17 sur 264$)$ & & \\
\hline
\end{tabular}


Tableau II (suite)

\begin{tabular}{|c|c|c|c|c|c|}
\hline \multirow{3}{*}{$\begin{array}{c}\text { Pays } \\
\text { États-Unis } \\
\text { États-Unis } \\
\text { États-Unis }\end{array}$} & \multirow{4}{*}{$\begin{array}{c}\text { Races } \\
\text { Brune des Alpes (Brown Swiss) } \\
\text { Pie Noire (Holstein Friesian) } \\
\text { Rouge sans cornes (Red Poll) } \\
\text { Pie Rouge (Simmental) } \\
\text { Marc II }\end{array}$} & \multicolumn{2}{|c|}{ Fréquence (\%) $C$} & Cas signalé & \multirow{3}{*}{$\begin{array}{l}\text { Ref } \\
039 \\
092\end{array}$} \\
\hline & & 7,14 & (1 sur 14$)$ & & \\
\hline & & & & 1 & \\
\hline Etats-Unis & & 7,04 & (5 sur 71$)$ & & 214 \\
\hline France & Montbéliard & 0,86 & ( 2 sur 232 ) & & 027 \\
\hline \multirow[t]{3}{*}{ France } & Limousin & 5,84 & ( 8 sur 137 ) & & 049 \\
\hline & Blonde d'Aquitaine & 19,61 & (10 sur 51$)$ & & \\
\hline & Charolais & 3,28 & (12 sur 366$)$ & & \\
\hline France & Non indiqué & & & 3 & 052 \\
\hline France & Blonde d'Aquitaine & $14,24($ & (302 sur 2121$)$ & & 053 \\
\hline France & Charolais & & & 1 & 054 \\
\hline France & Race Corse & 28,57 & (24 sur 84 ) & & 085 \\
\hline France & Montbéliard & & & 2 & 155 \\
\hline France & Montbéliard & 0,27 & (4 sur 1463 ) & & 162 \\
\hline France & Blonde d'Aquitaine & & & 3 & 166 \\
\hline \multirow[t]{4}{*}{ France } & Blonde d'Aquitaine & 10,71 & & & 172 \\
\hline & Limousin & 8,21 & & & \\
\hline & Montbéliard & 3,50 & & & \\
\hline & Charolais & 4,76 & & & \\
\hline \multirow[t]{2}{*}{ France } & Limousin & 0,41 & (2 sur 492 ) & & 173 \\
\hline & Pie Noire (Holstein Friesian) & & & & \\
\hline \multirow[t]{3}{*}{ France } & Limousin & 5,63 & (13 sur 231) & & 179 \\
\hline & Blonde d'Aquitaine & 20,00 & (45 sur 225 ) & & \\
\hline & Coopelso 93 & 28,57 & (8 sur 28$)$ & & \\
\hline France-Guadeloupe & Créole & & & 1 & 176 \\
\hline Grande-Bretagne & $\begin{array}{c}\text { Pie Rouge (Simmental) } \\
\text { Charolais }\end{array}$ & & & 2 & 030 \\
\hline Grande-Bretagne & Blanche Britannique (British White) & 78,86 & (41 sur 52 ) & & 036 \\
\hline Grande-Bretagne & Charolais & & & 1 & 087 \\
\hline Grande-Bretagne & Pie Rouge (Simmental) & 7,69 & (2 sur 26$)$ & & 089 \\
\hline \multirow[t]{2}{*}{ Grande-Bretagne } & Charolais & 0,54 & (1 sur 185 ) & & 091 \\
\hline & Pie Rouge (Simmental) & 2,65 & ( 3 sur 113 ) & & \\
\hline Grande-Bretagne & Charolais & & & 2 & 116 \\
\hline Grande-Bretagne & Blanche Britannique (British White) & 70,40 & & & 122 \\
\hline Grande-Bretagne & Pie Noire (Holstein Friesian) & 0,30 & $(1$ sur 330$)$ & & 154 \\
\hline Hongrie & Grise des Steppes & 3,77 & (4 sur 106$)$ & & 108 \\
\hline \multirow[t]{2}{*}{ Hongrie } & Pie Rouge (Simmental) & 2,88 & (16 sur 556$)$ & & 109 \\
\hline & Grise des Steppes & 9,09 & $(7$ sur 77$)$ & & \\
\hline Hongrie & Grise des Steppes & & & 1 & 110 \\
\hline Hongrie & Pie Noire (Holstein Friesian) & $\begin{array}{l}9,52 \\
1,64\end{array}$ & $\begin{array}{l}(6 \text { sur } 63) \\
(1 \text { sur } 61)\end{array}$ & & 138 \\
\hline Hongrie & $\begin{array}{l}\text { Pie Rouge (Simmental) } \\
\text { Pie Rouge (Simmental) }\end{array}$ & $\begin{array}{r}2,63 \\
25,00\end{array}$ & $\begin{array}{l}(2 \text { sur } 76) \\
(1 \text { sur } 4)\end{array}$ & & 146 \\
\hline Hongrie & Non indiqué & 18,18 & (2 sur 11$)$ & & 193 \\
\hline Italie & Podolian & 32,20 & (19 sur 59 ) & & 009 \\
\hline Italie & Romagnola & 22,07 & & & 040 \\
\hline Italie & Romagnola & 28,57 & & & 055 \\
\hline
\end{tabular}


Tableau II (suite)

\begin{tabular}{|c|c|c|c|c|c|}
\hline Pays & Races & \multicolumn{2}{|c|}{ Fréquence (\%) } & Cas signalé & Aef \\
\hline Italie & Brune des Alpes (Brown Swiss) & & & 1 & 060 \\
\hline Italie & Maremmana & & & 1 & 181 \\
\hline Italie & Romagnola & 50,00 & $(2$ sur 4$)$ & & 186 \\
\hline Italie & Chianina & & & 1 & 195 \\
\hline \multirow[t]{2}{*}{ Italie } & Chianina & & & 1 & 210 \\
\hline & Marchigiana & & & 1 & \\
\hline Italie & Romagnola & 22,22 & $(2$ sur 9$)$ & & 211 \\
\hline \multirow[t]{5}{*}{ italie } & Marchigiana & 18,75 & (3 sur 16$)$ & & 213 \\
\hline & Chianina & 9,52 & (2 sur 21 ) & & \\
\hline & Romagnola & 22,27 & (49 sur 220) & & \\
\hline & Modicana & 6,45 & $(2$ sur 31$)$ & & \\
\hline & Ottonese & 3,57 & (1 sur 28$)$ & & \\
\hline Japon & Noire Japonaise (Japanese Black) & 16,07 & (18 sur 112$)$ & & 082 \\
\hline Japon & Noire Japonaise (Japanese Black) & 12,73 & $(7$ sur 55$)$ & & 125 \\
\hline Japon & Noire Japonaise (Japanese Black) & & & 1 & 194 \\
\hline Maroc & Brune de l'Atlas & 25,00 & (1 sur 4$)$ & & 045 \\
\hline Maroc & Brune de l'Atlas & 8,26 & (9 sur 109$)$ & & 123 \\
\hline Nigeria & Red Bororo & 10,00 & $(1$ sur 10$)$ & & 147 \\
\hline Norvège & Pie Rouge (Simmental) & 4,18 & (18 sur 430$)$ & & 001 \\
\hline Norvège & Pie Rouge (Simmental) & 17,14 & (24 sur 140$)$ & & 183 \\
\hline \multirow[t]{2}{*}{ Nouvelle Zélande } & Blonde d'Aquitaine & & & 1 & 020 \\
\hline & Pie Rouge (Simmental) & & & 1 & \\
\hline Pologne & Charolais & 30,77 & (4 sur 13 ) & & 100 \\
\hline Pologne & Pie Noire (Holstein Frisonne) & & & 1 & 199 \\
\hline Pologne & Pie Rouge (Simmental) & & & 5 & 200 \\
\hline Pologne & Pie Rouge (Simmental) & & & 1 & 217 \\
\hline Portugal & Maronesa & 49,54 & (54 sur 109) & & 180 \\
\hline Roumanie & Pie Rouge (Simmental) & 1,50 & (3 sur 200$)$ & & 024 \\
\hline Roumanie & Pie Rouge (Simmental) & & & 1 & 025 \\
\hline Roumanie & Charolais & & & 1 & 113 \\
\hline Roumanie & Charolais & & & 1 & 189 \\
\hline Suède & Pie Rouge (Simmental) & 21,05 & (12 sur 57$)$ & & 035 \\
\hline Suède & Pie Rouge (Simmental) & 7,14 & (2 sur 28) & & 061 \\
\hline Suède & Pie Rouge (Simmental) & 14,33 & (293 sur 2045 ) & & 063 \\
\hline Suède & Pie Rouge (Simmental) & 14,00 & & & 064 \\
\hline Suède & Pie Rouge (Simmental) & 12,50 & (118 sur 944$)$ & & 067 \\
\hline Suède & Pie Rouge (Simmental) & & & 3 & 078 \\
\hline Suède & Pie Rouge (Simmental) & 14,50 & (sur 2000 ) & & 215 \\
\hline Suisse & $\begin{array}{l}\text { Pie Rouge (Simmental) } \\
\text { Brune des Alpes (Brown Swiss) }\end{array}$ & $\begin{array}{l}1,84 \\
0,40\end{array}$ & $\begin{array}{l}\text { (23 sur } 1251) \\
(4 \text { sur } 998)\end{array}$ & & 218 \\
\hline Tchécoslovaquie & Brune des Alpes (Brown Swiss) & & & 2 & 010 \\
\hline Tchécoslovaquie & Pie Rouge (Simmental) & 9,52 & (4 sur 42 ) & & 119 \\
\hline Tchécoslovaquie & Non indiqué & & & 1 & 196 \\
\hline Tchécoslovaquie & Pie Rouge (Simmental) & 5,70 & & & 197 \\
\hline Tchécoslovaquie & Pie Rouge (Simmental) & & & 1 & 198 \\
\hline Thaillande & Thaï & & & 2 & 044 \\
\hline URSS & Pie Noire (Holstein Friesian) & & & 1 & 008 \\
\hline URSS & Pie Noire (Holstein Friesian) & & & 1 & 058 \\
\hline URSS & Non indiqué & & & 1 & 059 \\
\hline URSS & Pie Rouge (Simmental) & 7,64 & (63 sur 825$)$ & & 101 \\
\hline
\end{tabular}


Tableau II (suite)

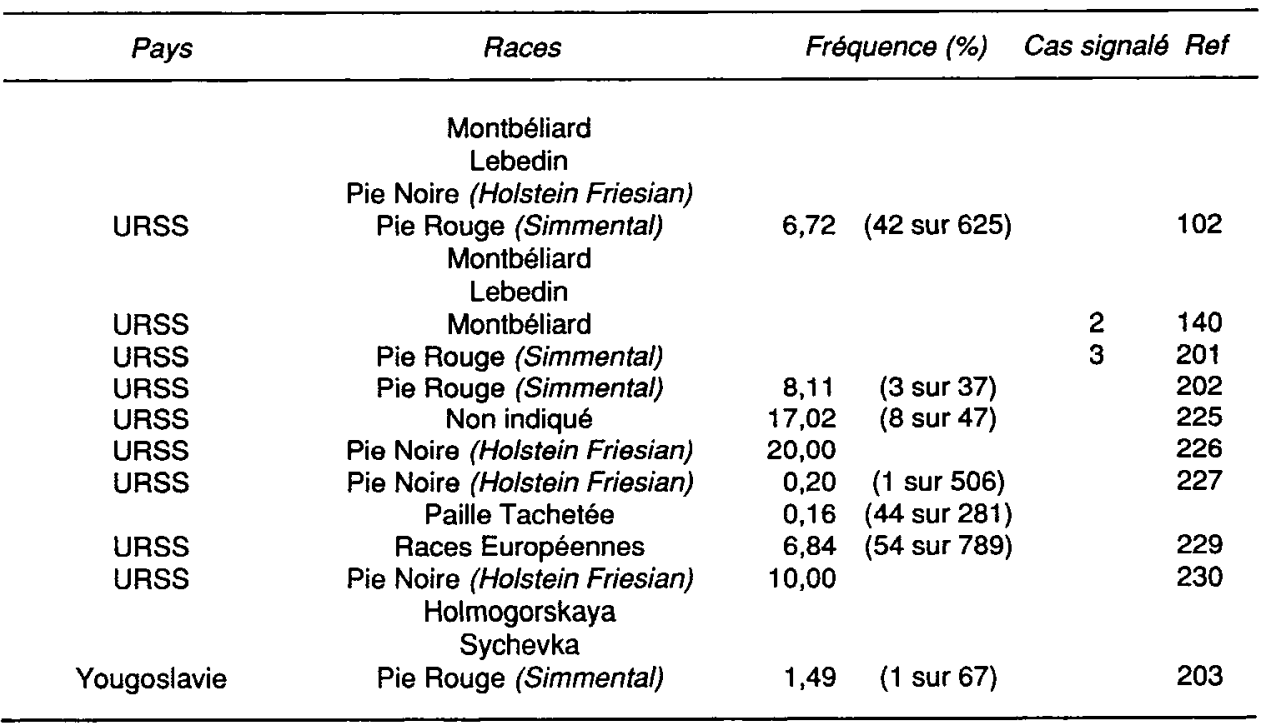




\section{Index races - pays}

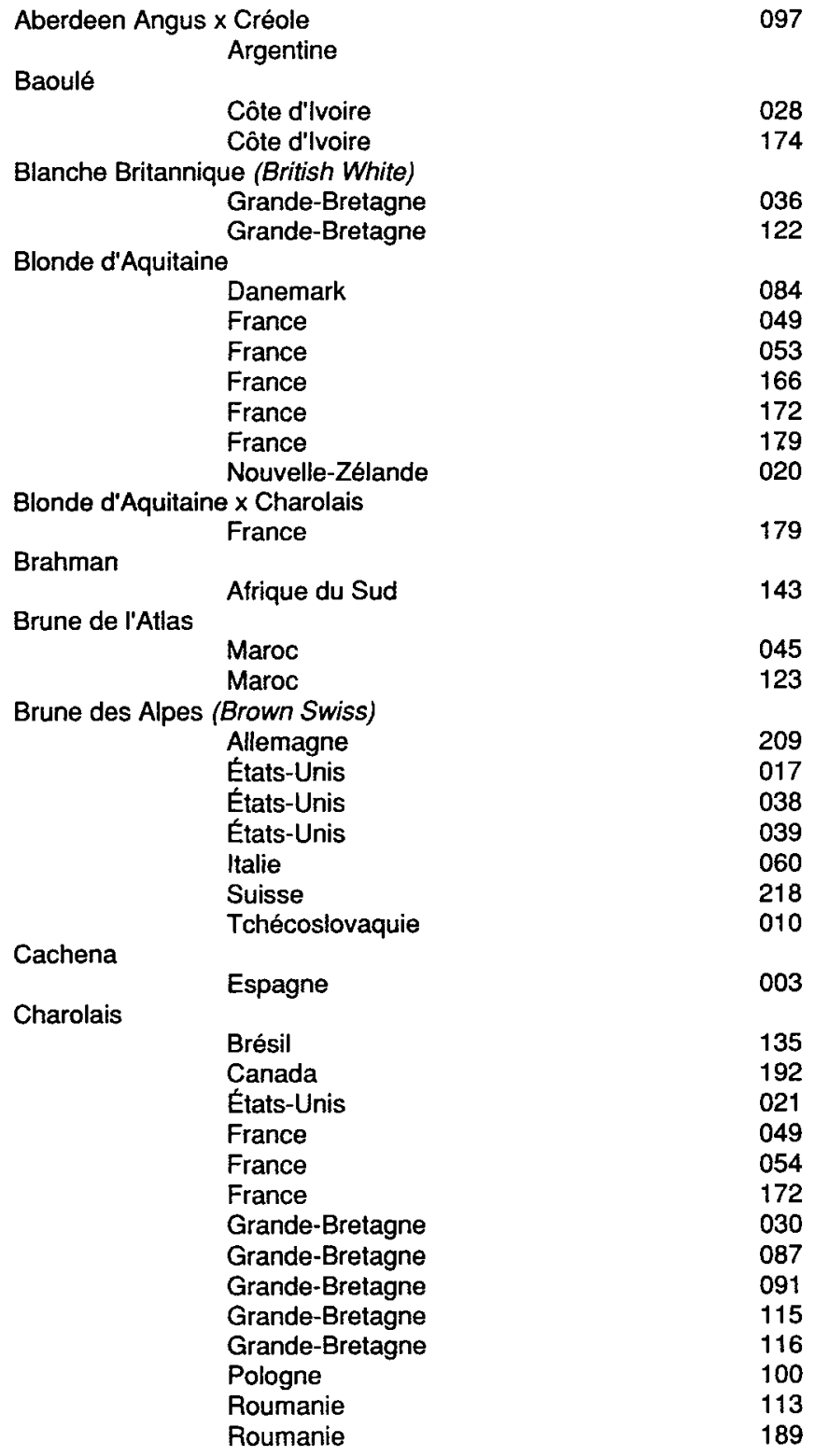


Charolais $x$ Jersey

Chianina

Afrique du Sud $\quad 086$

Brésil $\quad 043$

Italie $\quad 195$

Italie $\quad 210$

Italie $\quad 213$

Coopelso 93

$\begin{array}{ll}\text { France } & 179\end{array}$

Créole

France-Guadeloupe $\quad 176$

Grise Autrichienne (Tyrol Grey)
Autriche

Grise des Steppes

$\begin{array}{ll}\text { Hongrie } & 109\end{array}$

Guernsey

Hongrie $\quad 110$

Holmogorskaya

$\begin{array}{ll}\text { Canada } & 018\end{array}$

Jersey $x$ Charolais

URSS 230

Afrique du Sud $\quad 133$

Lebedin

URSS 101

$\begin{array}{ll}\text { URSS } & 102\end{array}$

Lidia

Espagne 003

Limousin

Espagne $\quad 224$

France $\quad 049$

France $\quad 172$

France $\quad 173$

France $\quad 179$

Limousin x Blonde d'Aquitaine

France $\quad 179$

Limousin $\times$ Charolais

France $\quad 179$

Limousin $\times$ Coopelso 93

France $\quad 179$

Marc II

Etats-Unis $\quad 214$

Marchigiana

Brésil 152

Italie $\quad 210$

Italie 213

$\begin{array}{lll}\text { Maremmana Italie } & 181\end{array}$

Maronesa $\quad$ Portugal 180

Modicana Italie 056

Italie $\quad 213$ 
Montbéliard

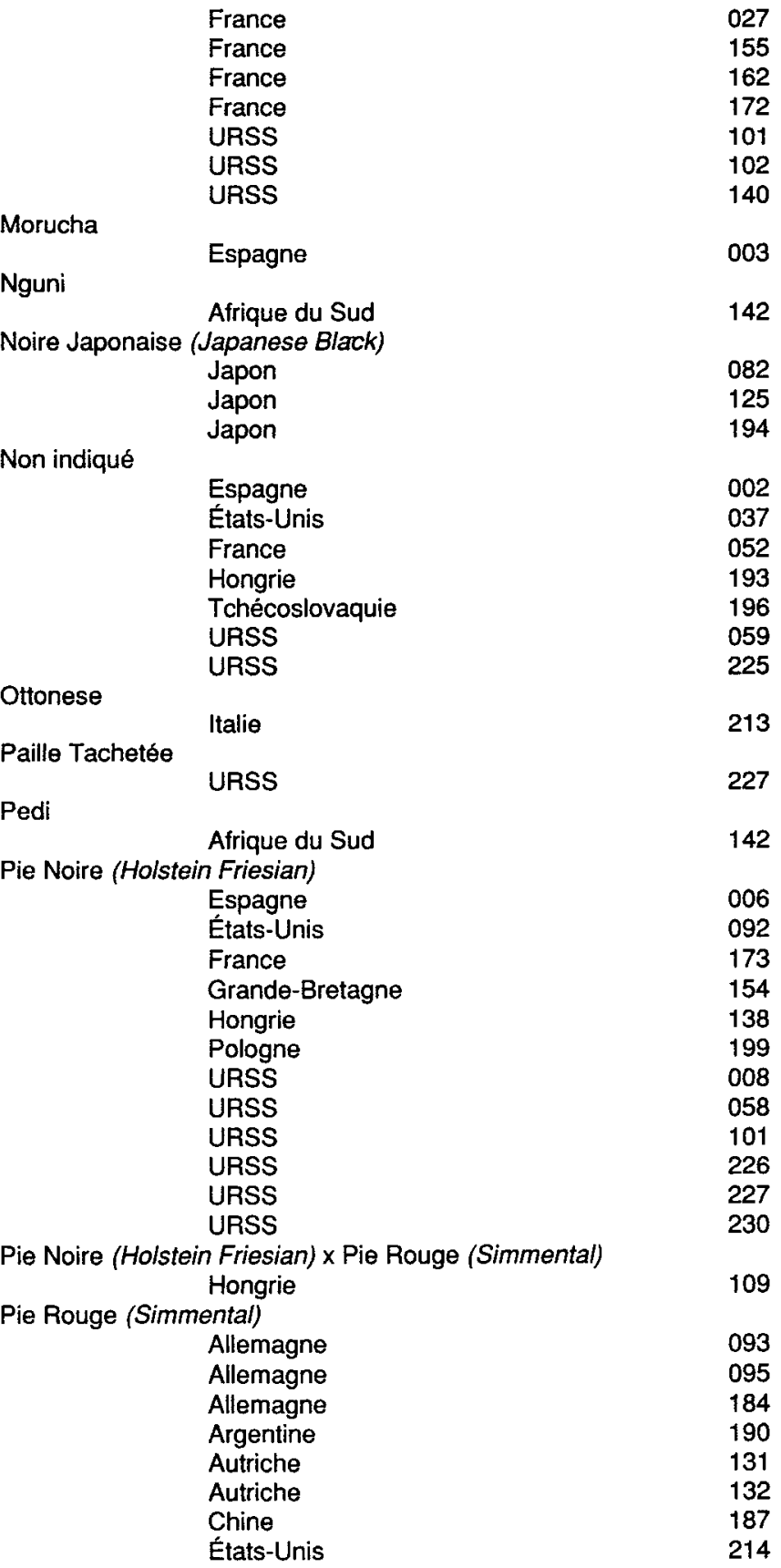


Grande-Bretagne 030

Grande-Bretagne $\quad 089$

Grande-Bretagne 091

Grande-Bretagne $\quad 115$

Hongrie $\quad 109$

Hongrie $\quad 146$

Norvège $\quad 001$

Norvège 183

Nouvelle-Zélande $\quad 020$

Pologne 200

Pologne 217

Roumanie 024

Roumanie 025

Suède 035

Suède 061

Suède 063

Suède $\quad 064$

Suède $\quad 067$

Suède $\quad 078$

Suède 215

Suisse 218

Tchécoslovaquie $\quad 119$

Tchécoslovaquie $\quad 197$

Tchécoslovaquie 198

URSS 101

URSS 102

URSS 201

URSS 202

Yougoslavie 203

Pie Rouge (Simmental) $\times$ Pie Noire (Holstein Friesian)
Pologne

Piebald

Allemagne

Pitangueiras

$\begin{array}{ll}\text { Allemagne } & 209\end{array}$

Brésil 136

Brésil 149

Brésil 150

Brésil 151

$\begin{array}{lll}\text { Podolian Italie } & 009\end{array}$

Race Corse France 085

$\begin{array}{ll}\text { Races Bavaroises Allemagne } & 047\end{array}$

Races Espagnoles

Espagne 005

Espagne $\quad 007$

Races Europénnes

URSS 229

$\begin{array}{lll}\text { Red Bororo } & \text { Nigeria } & 147\end{array}$

$\begin{array}{ll}\text { Retinta Espagne } & 003\end{array}$

Espagne 137 


\begin{tabular}{|c|c|c|}
\hline \multicolumn{3}{|l|}{ Romagnola } \\
\hline & Italie & 040 \\
\hline & Italie & 055 \\
\hline & Italie & 186 \\
\hline & Italie & 211 \\
\hline & Italie & 213 \\
\hline \multicolumn{3}{|c|}{ Rouge sans cornes (Red Poll) } \\
\hline & Australie & 081 \\
\hline & États-Unis & 214 \\
\hline & Grande-Bretagne & 115 \\
\hline \multicolumn{3}{|c|}{ Santa Gertrudis } \\
\hline & Cuba & 011 \\
\hline & Cuba & 015 \\
\hline & Cuba & 016 \\
\hline \multicolumn{3}{|l|}{ Sayaguesa } \\
\hline & Espagne & 003 \\
\hline \multicolumn{3}{|l|}{ Sychevka } \\
\hline & URSS & 230 \\
\hline \multicolumn{3}{|c|}{ Taureau de Combat } \\
\hline & Espagne & $\begin{array}{l}004 \\
223\end{array}$ \\
\hline \multicolumn{3}{|l|}{ Thaï } \\
\hline & Thaïlande & 044 \\
\hline \multicolumn{3}{|l|}{ Zebu Malawi } \\
\hline \multirow{3}{*}{ Zebu x N'Dama } & Afrique du Sud & 142 \\
\hline & Côte d'Ivoire & 028 \\
\hline & Côte d'Ivoire & 174 \\
\hline
\end{tabular}

